\title{
Vertical fluxes conditioned on vorticity and strain reveal submesoscale
}

\section{ventilation}

\author{
Dhruv Balwada*
}

School of Oceanography, University of Washington, Seattle, WA, USA

\section{Qiyu Xiao}

Courant Institute of Mathematical Sciences, New York University, New York, NY, USA

Shafer Smith

Courant Institute of Mathematical Sciences, New York University, New York, NY, USA

Ryan Abernathey

Lamont Doherty Earth Observatory, Columbia University, Palisades, NY, USA

Alison R. Gray

School of Oceanography, University of Washington, Seattle, WA, USA

${ }_{13}^{*}$ Corresponding author address: Dhruv Balwada, School of Oceanography, 1503 NE Boat Street,

${ }_{14}$ Box 357940, Seattle, WA 98195-7940

${ }_{15}$ E-mail: dbalwada@uw.edu 


\section{ABSTRACT}

It has been hypothesized that submesoscale flows play an important role ${ }_{17}$ in the vertical transport of climatically important tracers, due to their strong ${ }_{18}$ associated vertical velocities. However, the multi-scale, non-linear and La19 grangian nature of transport makes it challenging to attribute proportions of 20 the tracer fluxes to certain processes, scales, regions or features. Here we ${ }_{21}$ show that criteria based on the surface vorticity and strain joint probability ${ }_{22}$ distribution function (JPDF) effectively decomposes the surface velocity field ${ }_{23}$ into distinguishable flow regions, which roughly correspond to flow features ${ }_{24}$ like fronts and eddies. The JPDF has a distinct shape, and approximately

${ }_{35}$ but also enhances the flux due the larger scales. 


\section{Introduction}

Accurate projections of future climate depend crucially on our ability to constrain and predict the magnitude, distribution, and efficiency of oceanic uptake of heat, oxygen, carbon, and other important biogeochemical tracers. This tracer transport is influenced by flows at many scales. While the importance of mean flows and mesoscale eddies in transporting tracers has been recognized for many decades (Price et al. 1987; Marshall et al. 1993; Marshall 1997), recent evidence has suggested a significant contribution from submesoscale flows.

Submesoscale flows are characterized by Rossby $(R o)$ and and Richardson $(R i)$ numbers that approach unity, and are associated with lateral scales roughly an order of magnitude smaller than the first internal deformation radius. This deviation from geostrophy allows strong vertical velocities to develop. They are usually more active near a boundary, and can emerge from instabilities of a mean horizontal buoyancy gradient (Boccaletti et al. 2007; Fox-Kemper et al. 2008; Callies et al. 2016), stirring by mesoscale eddies (Hoskins and Bretherton 1972; Lapeyre and Klein 2006; Roullet et al. 2012), or by interactions of fronts with surface forcing (Thomas et al. 2008). Regardless of the generation mechanism, these scales play a dominant role in setting the mixed layer properties (Su et al. 2018), and are thought to be key in transporting tracers across the mixed layer base (e.g. Ferrari 2011; Lévy et al. 2018; Mahadevan et al. 2020; Uchida et al. 2020)

Observational evidence highlighting the strong vertical transport associated with individual submesoscale fronts has grown over the years (Omand et al. 2015; Adams et al. 2017; Olita et al. 2017; Ruiz et al. 2019; Archer et al. 2020; Siegelman et al. 2020), usually in the form of tracer filaments that are seen penetrating across the base of the mixed layer along an isopycnal, or as strong vertical velocities that extend far below the mixed layer base. However, observationally 
assessing the impact of these structures on regional and global scales remains challenging, due to a lack of statistical knowledge about their strength and frequency.

Modeling studies have suggested that resolving submesoscale flows quantitatively changes the tracer exchange across the mixed layer base. Such models include those that simulate single flow features like fronts or eddies (Mahadevan and Tandon 2006; Ramachandran et al. 2014; Brannigan 2016; Freilich and Mahadevan 2019), as well as those using large domains that are many deformation radii in size and represent a large region of the ocean (Lévy et al. 2001; Balwada et al. 2018; Klocker 2018; Uchida et al. 2019; Bachman and Klocker 2020). However, even given the complete spatio-temporal simulated data provided by models, the attribution of the enhanced vertical transport to specific submesoscale processes, dynamics, or scales is not straightforward.

The difficulty in attribution can be appreciated by considering the flow and tracer transport in the submesoscale-resolving simulation of Balwada et al. (2018, described in section 2a). The surface vorticity field clearly indicates the presence of submesoscale features like fronts and eddies (Figure 1a), and a passive tracer that is introduced at the surface reaches the interior in filaments and curtains that correspond visually with these features (Figure 1b). This correspondence results from two factors ${ }^{1}$ : the tracer is being injected into the interior in regions associated with the strong submesoscale filaments and fronts, and more importantly, once a tracer filament reaches sufficient depth, it gets stirred by the dominant horizontal flow associated with these features at that depth. It is important to note that the vertical velocity and tracer flux are highly variable: a snapshot of vertical velocity is largely dominated by high-frequency waves (Figure 1c), and the magnitudes of vertical flux in a snapshot (Figure 1d,e) are two orders of magnitude larger than

\footnotetext{
${ }^{1}$ Readers can also refer to the movies of the tracer field evolution in these simulations that are provided in the supplementary material of Balwada et al. (2018).
} 
its respective spatial average (Figure 1f). These properties suggest that a certain degree of spatiotemporal averaging is required to elucidate the vertical transport process.

A number of different approaches have been used to attribute transport to flow features or scales. The simplest, but coarsest, approach is Eulerian averaging over a spatial region that is primarily associated with the features of interest (e.g. Balwada et al. 2018; Klocker 2018; Freilich and Mahadevan 2019). Estimating the wavenumber and/or frequency cross-spectra of the vertical fluxes over a fixed region helps provide more insight by distinguishing the influence of different scales. For example, Balwada et al. (2018) compared spectra of vertical velocities and fluxes to show that internal waves, which dominate the vertical velocities, have a negligible impact on the tracer flux. However, this study also showed that the vertical flux has a broadband signal, with a wide range of scales contributing comparably. This broadband signal can be partially understood by noting that phase information is lost when plotting power spectra (Armi and Flament 1985; Franks 2005): a sharp front has a broadband signal in a power spectrum, instead of a sharp peak, and the shape of this spectrum is not sufficient to know that it represents a sharp front. Further, spectra of Eulerian fields may also suffer from Doppler shifting: a geostrophically balanced front being advected through a region by the mesoscale flow may have an imprint at the superinertial frequencies and suggest a lack of balance where none is present (Callies et al. 2020). Another approach is based on identifying coherent structures and estimating statistics following these structures. The simplest identification methods define structures based on some simple criteria (e.g. Capet et al. 2008, used a threshold on the second derivative to define fronts), while the most complex determine the structures using algorithms derived rigorously from continuum mechanics and dynamical systems theory (Haller 2015). The flow field is essentially cleaved into regions that are identified as coherent structures, under a particular selection criteria, and everything else. However, it is often found that the regions around but outside the structure boundaries are actually the most important for 
transport (Abernathey and Haller 2018; Zhang et al. 2020). Furthermore, in the presence of high frequency motions (e.g. inetria-gravity waves), it is often hard to even identify coherent structures (Sinha et al. 2019).

The objective of this work is to decompose the flow into distinct regions, which are roughly associated with fronts and eddies, and to quantify the role of these regions in the vertical transport of tracers. Our approach is motivated by Shcherbina et al. (2013), and centers on viewing the flow as a function of the surface vorticity and strain using a joint probability distribution function (JPDF). We find that different regions in the vorticity-strain space correspond roughly to distinct flow features, and conditional averaging allow us to distinguish the impact of these regions on vertical transport. We also find that the extent of the vorticity-strain JPDF is to some extent scale-selective, allowing also for the identification of flux with features of different scales. This technique is much simpler to implement than some of the coherent structure detection methods, and does not discard regions as not being part of a coherent structure.

This paper is organized as follows. In section 2 we briefly review the simulations that are analyzed here, and investigate the properties of the associated vorticity-strain JPDFs. In section 3, we consider the vertical velocities and fluxes of a passive tracer, demonstrating that the additional flux at higher resolutions is associated with small-scale fronts and the submesoscale-driven changes in properties of the large scale flows. We conclude and discuss further applications in section 4 .

\section{Flow structures in vorticity-strain space}

\section{a. Model details}

All the diagnostics and results presented in study are from the analysis of a series of simulations, using the MITgcm (Marshall et al. 1997), first presented in Balwada et al. (2018). The model 
setup is that of a channel forced by winds and thermal restoring, fashioned to be a simplified and idealized version of the Antarctic Circumpolar Current. The model domain is $2000 \mathrm{~km}$ by $2000 \mathrm{~km}$ horizontally and $3 \mathrm{~km}$ deep, with a Gaussian ridge that spans the entire meridional extent of the domain and is $1 \mathrm{~km}$ high (shallowest point in the domain is $2 \mathrm{~km}$ ) and $150 \mathrm{~km}$ wide (standard deviation of $75 \mathrm{~km}$ ). The model is set on a $\beta$-plane centered at $35^{\circ} \mathrm{S}$, and throughout the text, we use $f$ to indicate the meridionally-dependent Coriolis frequency, and $f_{0}=f\left(35^{\circ} \mathrm{S}\right)$. The surface forcing consists of a sinusoidal zonal wind stress akin to an atmospheric jet, with a single maximum in the center of the domain and zero at the boundaries, and a linear temperature restoring at the surface. Three different horizontal resolutions are used: $20 \mathrm{~km}, 5 \mathrm{~km}$ and $1 \mathrm{~km}$. The vertical grid is the same for all simulations, with 76 levels, $1 \mathrm{~m}$ spacing near the surface and approximately $150 \mathrm{~m}$ spacing near the bottom. The vertical diffusivity, $K$, is prescribed by the KPP scheme (Large et al. 1994). The vertical grid and numerics are the same as those in the LLC4320 simulations (e.g. Rocha et al. 2016).

After the model fields were spun up, a tracer was forced at the surface by restoring to a target value of $1 \mathrm{~kg} \mathrm{~m}^{-3}$ in the top $1 \mathrm{~m}$ grid cell, with a restoring time scale of 72 minutes. This restoration rate corresponds to gas transfer velocity of $80 \mathrm{~cm} \mathrm{hr}^{-1}$, which is similar to observed values under moderate to high wind conditions in the Southern Ocean (Ho et al. 2006). See Balwada et al. (2018) for more details on the model setup, spectral properties of the simulations, and an analysis of the influence of horizontal resolution on the tracer uptake.

The tracer is continually forced at the surface for one year, and the amount of the tracer in interior increases throughout this year. Most of the analysis in this study is done using snapshots of the flow field separated by 10 days, and spread over this year. After the tracer is switched on, the tracer concentration undergoes a transient phase of about 2 months during which it is taken up rapidly in the mixed layer by boundary layer diffusion. During the period between months $3-12$, the 
tracer concentration in the interior is increasing, but the fluxes at the surface and base of the mixed layer stay in relative equilibrium. Model output for the diffusive fluxes of tracers were only saved for the first 6 months of the simulation, which limits the analysis period that can be considered when analyzing tracer budgets. Therefore, in section 3b we use daily snapshots from months 3-6. While for other conditional averages in this study we averaged over 30 time snapshots of model output, taken every 10 days (months 3-12). However, none of the statistical results in this study are qualitative effected by these choices of the number and frequency of snapshots used, since we ensured that all the statistics evaluated are converged. Spatially, the analyzed region extends from $y=500 \mathrm{~km}$ to $y=1500 \mathrm{~km}$ (Figure $2 \mathrm{a}$ ), which excludes regions adjacent to the northern and southern boundaries to ensure that unrealistic dynamics due to the presence of vertical walls do not influence the results.

\section{b. Joint probability distribution function (JPDF) of vorticity and strain}

The analysis of two-dimensional flows in terms of the gradients of the velocity field (the strain tensor) is a fundamental tool with a long history (Okubo 1970; Weiss 1991). This serves as partial motivation for our investigation, with the acknowledgement that the surface flow can have significant deviations from a two-dimensional flow. This analysis is reviewed in Appendix A, and there it is also shown that the velocity gradient tensor can be expressed in terms of the vertical vorticity, horizontal divergence, normal strain and shear strain. These are defined respectively as

$$
\zeta=v_{x}-u_{y}, \quad \Delta=u_{x}+v_{y}, \quad \sigma_{n}=u_{x}-v_{y}, \quad \text { and } \quad \sigma_{s}=v_{x}+u_{y}
$$

The normal and shear strains are not coordinate-invariant, however the vorticity, divergence, and strain magnitude

$$
\sigma=\sqrt{\sigma_{n}^{2}+\sigma_{s}^{2}}
$$


are. Unless noted otherwise, the term 'strain' will correspond to the strain magnitude normalized by the absolute value of the Coriolis frequency $\sigma /\left|f_{0}\right|$, the 'vorticity' to the vorticity normalized by the Coriolis frequency $\zeta / f_{0}$, and 'divergence' to the divergence normalized by the absolute value of the Coriolis frequency $\Delta /\left|f_{0}\right|$.

Snapshots of vorticity, strain, and tracer concentration at three resolutions, presented in Figure 2, clearly show the presence of coherent features, with the visually prominent features becoming smaller in size and stronger in magnitude as resolution is increased. In fact, the coherent features roughly correspond to distinct signatures in vorticity and strain: cyclones have a prominent high vorticity core and a weak imprint on strain, fronts are associated with high-vorticity and high-strain filaments, and so forth. The asymmetry in the vorticity field is also clear at higher resolutions: the vorticity map is composed of a broad but relatively weak negative vorticity soup punctuated with sharp and long positive vorticity filaments and vortices. Furthermore, the imprint of these flow features on the tracer is clear, even below the mixed layer.

The distinct signature of different flow features on vorticity, strain, and tracer concentration suggests that a statistical approach may reveal more quantitative connections. Inspired by results presented in Shcherbina et al. (2013), we consider the joint probability distribution function (JPDF) of surface vorticity and strain,

$$
P(\zeta, \sigma), \quad \text { where } \iint P(\zeta, \sigma) \mathrm{d} \zeta \mathrm{d} \sigma=1,
$$

and the conditional means of different variables conditioned on the surface vorticity and strain $\left(\overline{(.)^{\zeta \sigma}}\right)$. See Appendix B for the details of JPDF and conditional mean calculation for discrete ranges and finite data.

The JPDF has a distinct shape (Figure 3); it is centered near the origin, extends along lines of $\sigma=|\zeta|$, and is skewed with a longer cyclonic tail. This shape is a robust feature, and has been 
previously noted in other numerical simulations (Shcherbina et al. 2013; Rocha et al. 2016), as well as in real oceanic flows (Shcherbina et al. 2013; Berta et al. 2020). This shape probably arises from a balance between the strength of the large-scale instabilities energizing the mesoscale eddying flow, which produces a cascade of gradients to smaller scales, and the smaller-scale instabilities and dissipation halting the growth of gradients at the finest scales (discussed further in appendix C). Thus, the exact extent of the JPDF might vary regionally and seasonally (Rocha et al. 2016).

In this study, we decompose the regions of the JPDF and thus correspondingly the flow field into three parts or regions, corresponding to anticyclonic vorticity dominated (AVD) regions $(\zeta<0 \&$ $\sigma<|\zeta|)$, cyclonic vorticity dominated (CVD) regions $(\zeta>0 \& \sigma<|\zeta|)$, and strain dominated (SD) regions $(\sigma \geq|\zeta|)$

The flow decomposition for a snapshot from the $1 \mathrm{~km}$ simulation in a sub-region (delineated by the dashed square in Figure 2a) is considered as an example, and highlights a rough correspondence with coherent flow features (Figure 4). The flow here is composed of a large anticyclonic swirl, embedded with fronts and cyclones. The reliability of our ad hoc separation in roughly parsing flow features is supported by plotting separately the vorticity in $x-y$ space corresponding to the AVD, CVD, and SD regions. As expected, the panel corresponding to the AVD region shows the presence of a large anticyclonic swirl, the CVD region shows the presence of small intense cyclones, and the SD region shows filamentary vorticity streaks that correspond to fronts.

\section{c. Signatures of fronts}

Strain dominated (SD) regions are ubiquitous in the ocean and occupy the largest fraction of the surface area in the simulations (approximately 60\%); SD regions that are also associated with strong gradients in buoyancy (Figure $\mathrm{C} 1 \mathrm{~b}$ ) are referred to as fronts (e.g. Figure 5 shows the structure of a relatively straight front from the $1 \mathrm{~km}$ simulation). At fronts, the vertical velocities can 
coherently and adiabatically connect the mixed layer and the interior if the front is deep (Figure 5e), thus making them central in our study. Here we describe the canonical structure of fronts and try to better understand how they map onto the vorticity-strain JPDF.

During the process of frontogenesis, when a background flow is causing the surface density gradient to increase, an ageostrophic secondary circulation develops with a tendency to restratify the front: upwelling on the lighter side and downwelling on the heavier side. Typical submesoscale fronts tend to be asymmetric, with stronger cyclonic vorticity, convergence, and vertical velocity on the downwelling side of the front (Thomas et al. 2008; Shcherbina et al. 2013), as is evident in Figure 5 b,f. This asymmetry arises due to the vorticity tendency, $\partial_{t} \zeta=(f+\zeta) \partial_{z} w+\ldots$, having an asymmetric response to vortex stretching (McWilliams 2016). The vortex stretching near the surface strengthens the cyclonic vorticity, and compression strengthens the anticyclonic vorticity, but when $R o \sim O(1)$ the cyclonic vorticity strengthens more rapidly. Additionally, inertial instability also limits the range of anticyclonic vorticity that can be sustained (discussed in Appendix C).

The downwelling velocities at fronts can be very strong, $10-100 \mathrm{~m} /$ day, and have the potential to rapidly transport tracers to depth. We see signatures of this in Figure 5g,h, which shows that at the front the tracer penetrates as filaments to a few $100 \mathrm{~m}$ over the course of two days. In the particular case considered here, the tracer filament is not always perfectly aligned with isopycnals, which highlights the three-dimensionality of the transport process, and is likely a result of alongfront variations. The upwelling side of the front — with largely upwards vertical velocity — is also a site where deeper water is brought to the surface, as highlighted by tracer-free anomalies sliding upward along the front into the mixed layer.

Fronts generally have a strain magnitude (Figure 5c) that is greater than the vorticity. This can be explained by considering an ideal straight front and a local coordinate system oriented such 
that the along-front velocity points in the meridional direction, consider a velocity of the form $\mathbf{u}=0 \hat{\boldsymbol{x}}+v(x, z) \hat{\boldsymbol{y}}$. Then $y$-derivatives vanish, and definitions in (1) and (2) can be combined to show that

$$
\sigma^{2}=\Delta^{2}+\zeta^{2}>\zeta^{2}
$$

This suggests that in the vorticity-strain JPDF (Figure 5d) the ideal front will lie around or above the $\sigma=|\zeta|$ lines, at a distance that is determined by the strength of the surface divergence.

In the Hoskins and Bretherton (1972) classical theory of frontogenesis, with scaling for the atmospheric mesoscale, the associated ageostrophic divergence is small compared to the jet's vorticity and strain. Barkan et al. (2019) has however revisited this problem using asymptotic theory appropriate to submesoscale frontogenesis in the ocean's well-mixed surface layer. They show that when fronts are in turbulent thermal wind balance (TTWB, Gula et al. 2014), with $R o \sim O(1)$, the associated ageostrophic divergence scales like the vorticity and strain, i.e. $|\Delta| \sim|\zeta| \sim \sigma$, which for ideal fronts (equation 4) would correspond to points further up in the SD region of the JPDF. This oceanic regime, where the divergence is comparable in strength to strain and vorticity, is present in our simulation. The conditional mean divergence $\left(\bar{\Delta}^{\zeta \sigma}\right.$, Figure 6a) highlights the presence of rapid convergence in SD regions. We also consider a 3D JPDF of strain-vorticitydivergence, presented as a series of slices at various values of divergence in Figure 6b. Surface flows with the strongest convergence and divergence, $\Delta /\left|f_{0}\right| \sim O(1)$, lie almost exclusively in the SD region, in contrast to AVD and CVD regions having comparable instances of convergence and divergence that cancel each other out in the mean. This exclusive association between SD regions and the strongest surface convergence and divergence is suggestive that these regions might have an outstanding impact on vertical tracer fluxes. We confirm this hypothesis in section 3 . 


\section{d. Scale dependence in vorticity-strain space}

Regions of larger strain and vorticity are usually associated with smaller scales, a result of the forward cascade of enstrophy. Since the smaller scales are not resolved in lower resolution simulations, we expect that the range of vorticity and strain values sampled will decrease with resolution. This is confirmed by comparing the JPDFs from the simulations at different resolutions. The upper row of Figure 7 shows the JPDFs of vorticity and strain for the $5 \mathrm{~km}$ and $20 \mathrm{~km}$ simulations. Superposed on each figure are the outer contours of the JPDFs from the lower-resolution simulations, making it clear that the extent of the JPDF shrinks in size as resolution is lowered.

An alternative way to compare across scales is to use a coarse-graining filter on the highest resolution simulation. We specifically define a scalar field coarse-grained to grid-scale $h$ as

$$
\langle F\rangle^{h}\left(x_{i}, y_{j}\right) \doteq h^{-2} \int_{x_{i}-h / 2}^{x_{i}+h / 2} \int_{y_{j}-h / 2}^{y_{j}+h / 2} F(x, y) \mathrm{d} x \mathrm{~d} y .
$$

The coarse-grained vorticity is computed using the coarse-grained velocities as $\zeta^{h} \doteq \partial_{x}\langle v\rangle^{h}-$ $\partial_{y}\langle u\rangle^{h}$, and analogously for the coarse-grained strain $\sigma^{h}$. The velocities on the C-grid from MIT$\mathrm{gcm}$ were linearly interpolated to the tracer point before coarse graining, and then linearly interpolated to the lower resolution C-grid to compute finite difference gradients. This makes sense, since we want to compare the coarse-grained flow field to the flow field from a lower resolution simulation.

The bottom row of Figure 7 shows the JPDFs of vorticity and strain for the $1 \mathrm{~km}$ simulation, coarse-grained to $5 \mathrm{~km}$ (panel c) and $20 \mathrm{~km}$ (panel d). Remarkably, we see that the coarse-graining procedure shrinks the extent of the JPDF to almost exactly the contours for the lower resolution simulations. We tried a few different filtering techniques, and found that this qualitative result holds regardless of the exact methodology. This tells us that as resolution is increased and more submesoscale activity is admitted, the associated high strain and vorticity values come from fea- 
tures that are too small to resolve at lower resolution. Therefore, level-set contours of the JPDF of vorticity and strain can also be used as proxies for contours of lateral scales of flow features.

We use these ideas to segment the JPDF, and consequently the surface flow, beyond the SD, AVD and CVD regions. We subdivide the SD region from the $1 \mathrm{~km}$ simulation into a part of the $1 \mathrm{~km}$ JPDF that is also contained inside the extent of the JPDF of the $5 \mathrm{~km}$ resolution simulation (the region above the dashed lines and contained within the intermediate gray contour in Figure 3), and the part outside it (the region above the dashed lines and between within the outermost and intermediate gray contours in Figure 3). We will refer to these regions as $\mathrm{SD}^{>5 \mathrm{~km}}$ and $\mathrm{SD}^{<5 \mathrm{~km}}$ regions respectively, and will correspond roughly to mesoscale SD (resolvable at $5 \mathrm{~km}$ ) and submesoscale SD (only resolvable at $1 \mathrm{~km}$ ) regions. It is worth noting, that while the SD region in the $1 \mathrm{~km}$ simulation corresponds to approximately $60 \%$ of the spatial area, the $\mathrm{SD}^{<5 \mathrm{~km}}$ region represents less than $1 \%$ of the spatial area. We will use this decomposition and the level-set contours of the vorticity-strain JPDF more generally to parse the fluxes as an approximate function of scale in section $3 b$.

\section{Vertical velocities and tracer transport in vorticity-strain space}

Here we turn to the main theme of the paper: how to best determine what regions and structures are responsible for the vertical tracer exchange and how they change as resolution is increased. Having established in the previous sections how flow structures and scales are revealed in vorticitystrain space, we now consider vertical transport in this frame. We first consider vertical velocities conditioned on vorticity and strain, and then go on to study the impact of different regions in vorticity-strain space on the transport of a tracer that is restored at the surface. 


\section{a. Vertical velocities}

The conditional mean of vertical velocity $\left(\bar{w}^{\zeta \sigma}\right)$, Figure 8 , shows a pattern that is reminiscent of the conditional mean of surface divergence, Figure 6. This pattern is very robust, and emerges qualitatively even if a single snapshot is used, suggesting that the degree of spatial averaging that is implicit when estimating averages conditioned on vorticity and strain is sufficient to filter out the high-frequency wave field that dominates a spatial map of vertical velocity (as seen in Figure 1c), and provides a robust method to obtain the signal that is relevant for transport.

The conditional mean vertical velocities in the CVD and AVD regions are similar across resolutions - broadly AVD regions have upwelling in the mixed layer and downwelling below the mixed layer, while CVD regions are the opposite with downwelling near the surface and upwelling deeper down. The SD regions for the two lower resolution simulations are similar, with downwelling on the cyclonic side and upwelling on the anticyclonic side, and this pattern does not vary significantly down to a few $100 \mathrm{~m}$ below the mixed layer. This suggests that the fronts at these resolutions are relatively symmetric, and easily reach below the mixed layer base. In contrast, the $\mathrm{SD}$ region in the $1 \mathrm{~km}$ simulation is far from being symmetric and shows significant changes with depth. Most of the SD region at $1 \mathrm{~km}$ is characterized by downward velocities, with the upward velocities present only very close to the $\zeta=-\sigma$ line. The strongest downwelling is in regions farther away from the $\zeta=\sigma$ line, where the surface divergence is the strongest (compare with Figure 6).

Why do we find downwelling on the anticyclonic side of the SD region (likely associated with the warm side of fronts), and why does this occur only at the highest resolution? A plausible explanation comes from contrasting the secondary circulation associated with 2D frontogenesis in the quasi-geostrophic (QG) vs. semi-geostrophic (SG) equations (compare figures 1.8 and 1.9 
in Shakespeare 2015). QG frontogenesis is symmetric, and even as the front steepens in time, the vertical velocities change sign across the core of the front at all depths. In contrast, SG frontogenesis is not symmetric: the cyclonic side of the front sharpens rapidly, and the region of downwelling velocity, which is concentrated and strong on this cyclonic side near the surface, decreases in strength but widens laterally at depth to occupy part of the region that is under the warm/anticyclonic side of the frontal core. The frontogenesis at the lower resolutions, characterized by lower $R o$ and higher $R i$, is bound to be more akin to QG dynamics, while at the $1 \mathrm{~km}$ resolution the frontogenesis is likely better-described by SG dynamics.

\section{b. Vertical tracer transport}

Having considered how vertical velocities vary in different vorticity-strain regions, we now study how the different regions work in unison to transport a tracer from the surface into the interior. The tracer, $C$, in a control volume bounded horizontally over a geographical area and vertically from the sea floor to an arbitrary fixed depth $(z)$ evolves approximately according to the equation

$$
\partial_{t}\langle C\rangle^{z}=-\overline{w C}+\overline{K \partial_{z} C}+\bar{F} \delta(z)
$$

where $\overline{(.)}$ is the horizontal spatial mean at constant $z$ (refer to Appendix B), $\langle C\rangle^{z}=\int_{-H}^{z} \bar{C} \mathrm{~d} z^{\prime}$ is the total amount of tracer in the control volume divided by the horizontal domain area $A$, and $\delta(z)$ is the Dirac delta function that is non-zero only at the surface. The total amount of tracer below a given depth can increase due to the advective flux $(-\overline{w C})$, diffusive flux $\left(\overline{K \partial_{z} C}\right.$, where $K$ is prescribed by the KPP scheme and changes as a function of the flow), or surface flux (which is either zero, or $\bar{F}$ if the control volume extends all the way to the surface). The horizontal fluxes are ignored because they are small over the chosen domain (shown in Balwada et al. 2018), since there are are no lateral gradients in tracer restoring. 
In the mixed layer, both the advective and diffusive fluxes contribute, while below the mixed layer only the advective flux is non-zero. Balwada et al. (2018) found that during the initial few months of tracer forcing, the mixed layers are rapidly saturated with tracer, after which a quasisteady state is achieved. During this quasi-steady state, the surface flux does not change much, i.e. $\partial_{t}\langle C\rangle^{0}=\bar{F}$ is approximately constant, and thus even below the mixed layer, where the diffusive flux is zero, the rate of tracer change, $\partial_{t}\langle C\rangle^{z}=-\overline{w C} \approx \bar{F}$, is thus also approximately constant. The analysis presented here is for this phase of the tracer simulation.

\section{1) MEAN TRACER FLUXES CONDITIONED ON VORTICITY AND STRAIN}

The conditional means of fluxes show that each flux term is impacted by the different regions in very different ways (Figure 9). The conditional mean of the vertical advective tracer flux ( $\overline{w C}^{\zeta \sigma}$ ) near the base of the mixed layer (Figure 9a) is large and downward in the regions of rapid downwelling associated with SD regions, and upwards in regions of upwelling. In fact, it closely resembles the conditional mean of vertical velocities at the base of the mixed layer (compare to Figure 8b).

What does it mean to have upward advective tracer flux, when the tracer source is at the surface and the tracer is being fluxed downward by design? This can be understood by considering two things. First, since the tracer concentration is always positive, $C \geq 0$, regions of upwelling will necessarily have a positive flux, and only the spatial mean over the horizontal domain, $\overline{w C}$, need be downward (negative). Secondly, even if the flux is upwards, it does not imply that it will lead to an increase in tracer concentration above the depth level under consideration: the upward flux typically brings up waters with negative tracer anomalies (the concentration at depth is usually smaller than that in the shallower region). 
An alternate way to consider the advective flux is to consider its Reynold's decomposition

$$
w C=\bar{w} \bar{C}+\bar{w} C^{\prime}+w^{\prime} \bar{C}+w^{\prime} C^{\prime},
$$

where the eddy terms are defined relative to the spatial mean, as $C^{\prime}(x, y)=C(x, y)-\bar{C}$. The vertical advective flux or the total advective flux is composed of four components, where the second and third vanish when integrating over the domain, and the first term is negligible because the mean vertical velocity is very small. The last term, the vertical eddy advective flux, dominates the spatial mean of the advective flux. The difference between the conditional means of the total $\left(\overline{w C}{ }^{\zeta \sigma}\right)$ and eddy advective $\left(\overline{w^{\prime} C^{\prime}} \zeta \sigma\right)$ fluxes results from the third term $\left(w^{\prime} \bar{C}\right.$, not shown), which dominates the pattern of the conditional mean of the total advective flux and has a similar pattern to the conditional mean vertical velocity (Figure 8) but will make no contribution to the spatial mean of the eddy advective flux. In fact, the conditional mean eddy advective flux is downward (negative) almost everywhere in vorticity-strain space and is the strongest in the $\mathrm{SD}^{<5 \mathrm{~km}}$ region, supporting the idea that submesoscale fronts play an important role in net tracer ventilation (Figure 9b).

The transit of the tracer from the atmosphere to the ocean interior starts at the surface and proceeds through the mixed layer, so it is worth considering whether the different flow regions impact the surface and diffusive fluxes differently. The surface flux is high in regions of surface divergence (Figure 9c, compared with Figure 6), usually associated with the upwelling side of fronts and anticyclones. These are the regions where deeper low-tracer waters are pulled up to the surface, creating the strongest mixed layer tracer anomalies and thus the largest surface flux from a restoring condition. The surface flux is also large in regions of strong downwelling and surface convergence (around $0<\zeta / f_{0}<2$, compare to figure $6 \mathrm{~b}$ ). The variations of the conditional mean of the diffusive flux are similar to that for the surface flux (Figure 9d), with slightly weaker fluxes in the the SD regions with upward velocities. This is potentially a result of the upwelling bringing 
deeper stratified water into the mixed layer, and likely suppressing the mixing by KPP. Finally, it is worth noting that even though there are regional variations of the surface and diffusive fluxes, they are much weaker than the advective fluxes (notice the colorbars are logarithmic panels a,b and linear in panels c,d of Figure 9).

\section{2) NET TRACER FLUXES CONDITIONED ON VORTICITY AND STRAIN}

The conditional means considered above help understand the relative roles played by different flow regions, on average and in isolation. To understand the net contribution on tracer transport, we must consider the conditional mean of the fluxes in different regions along with the frequency of occurrence (via the JPDF $P(\zeta, \sigma)$, defined in equation 3 and appendix B). For example, the net contribution of advective flux as a function of vorticity and strain is $\widetilde{w C}=\overline{w C}^{\zeta \sigma} P(\zeta, \sigma)$, which if integrated over the whole vorticity-strain space would give the spatial integral of the advective flux, $\iint_{A} w C d x d y=\iint_{R} \overline{w C}^{\zeta \sigma} P(\zeta, \sigma) \mathrm{d} \zeta \mathrm{d} \sigma$.

The conditional mean of each flux component shows variations across regions, but as noted above these variations are much smaller for the surface and diffusive fluxes as compared to the advective flux. This results in the net impact of the surface and diffusive fluxes having variations across the vorticity-strain space that are set primarily by the variations in the JPDF (Figure 10c,d).

However, the conditional mean of advective fluxes varies by orders of magnitude across the vorticity-strain space, and its sum in different parts of the vorticity-strain space is not predominantly a function of the spatial surface area occupied by that part (Figure 10a,b). It is particularly noteworthy that this relatively higher contribution at the finer scales is primarily limited to the SD region. 
3) DEPTH DEPENDENCE OF THE TRACER FLUX CONTRIBUTION FROM DIFFERENT VORTICITYSTRAIN REGIONS

In Figure 11 we investigate the depth dependence of the contribution from the different flux components, integrated over different regions of the vorticity-strain JPDF, for the $1 \mathrm{~km}$ and $5 \mathrm{~km}$ resolution simulations. The surface flux matches the diffusive flux at the surface (Figure 11b,e) because the advective flux is zero here.

The advective fluxes (Figure 11a,d) are largest in the mixed layer, where a large cancellation between the SD and AVD regions takes place, while the contribution from the CVD region is relatively weak. The contribution from the AVD region rapidly diminishes below the base of the mixed layer, while the contribution from the SD region penetrates much deeper. This results in the sum of the advective fluxes peaking at the base of the mixed layer, and being primarily dominated by the SD regions at depths below the base of the mixed layer. Correspondingly, the eddy advective flux (Figure 11c,f) peaks at the base of the mixed layer and has the largest contribution from the SD region, with the AVD and CVD regions having a much smaller contribution and a weak depth dependence.

To compare the SD region between the $1 \mathrm{~km}$ and $5 \mathrm{~km}$ simulations, we separate the SD region into the smaller scale $\left(\mathrm{SD}^{<5 \mathrm{~km}}\right)$ and larger scale $\left(\mathrm{SD}^{>5 \mathrm{~km}}\right)$ regions, as described at the end of section $2 \mathrm{~d}$. The $\mathrm{SD}^{>5 \mathrm{~km}}$ region contributes more than the $\mathrm{SD}^{<5 \mathrm{~km}}$ region to the advective tracer flux (Figure 11a), as it occupies a much larger spatial area (SD ${ }^{>5 \mathrm{~km}}$ occupies approximately $59 \%$ of the area, while $\mathrm{SD}^{<5 \mathrm{~km}}$ occupies less than $1 \%$ ). The eddy advective flux near the base of the mixed layer in the $1 \mathrm{~km}$ simulation increased by about $40 \%$ relative to the $5 \mathrm{~km}$ simulation (compare Figure $11 \mathrm{c}$ to f), about $30 \%$ of this increase came from the $\mathrm{SD}^{>5 \mathrm{~km}}$ region and about $10 \%$ from the $\mathrm{SD}^{<5 \mathrm{~km}}$ region. This suggests that the enhanced tracer flux at higher resolutions is not only 
a result of additional tracer fluxes at small-scales being resolved, but also due to the contrubution from the large-scales increasing in response to the resolved small-scale flows. Balwada et al. (2018) and Uchida et al. (2019) reached a similar conclusion using spectral decompositions.

\section{4) Net CONTRIBUtion TO TRACER FLUX By DIFFERENT LATERAL SCALES}

The highest probability, the peak of the JPDF $P(\zeta, \sigma)$ in Figure 3, is near the origin and corresponds to vorticity and strain values at the largest scales and resolvable at all resolutions. The probability decreases as we move from the origin to higher vorticity and strain values, which correspond to smaller scales only resolved at higher resolutions (see also discussion in Section 2d). We form a new axis, $p_{\max } / p$, that takes a value of one when the particular probability value $(p)$ is the largest probability ( $p=p_{\max }$ ) near the origin, and extends to larger values outwards from the origin; this axis serves as a rough proxy for length scales. Here we consider the cumulative integrals of different properties as a function of $p_{\max } / p$, which for some property $\tilde{F}(\zeta, \sigma)$ (refer to appendix B for further details of notation) can be expressed as

$$
\breve{F}\left(p_{\max } / p\right)=\iint_{R} \tilde{F}(\zeta, \sigma) H[P(\zeta, \sigma)-p] \mathrm{d} \zeta \mathrm{d} \sigma,
$$

where $H(x)$ is the Heaviside function, which is 1 when $x \geq 0$ and 0 when $x<0$. As $p_{\max } / p \rightarrow \infty$, $\breve{F}$ corresponds to the area integral.

The different flux components add up at different rates as $p_{\max } / p$ increases, as shown for the $1 \mathrm{~km}$ simulation in Figure 12a. As discussed above, the surface and diffusive fluxes are relatively homogeneous compared to the advective fluxes, and asymptote to their total contributions at a rate that is set largely by how much spatial area is contained inside each $p_{\max } / p$ contour. This can be seen when comparing the area fraction to the flux fraction inside each $p_{\max } / p$ (Figure 12b).

In contrast, the eddy advective flux asymptotes much more slowly, clearly indicating that smaller scales - the points on the periphery of the JPDF, with larger $p_{\max } / p$ - play an outsized role. For 
example, the region outside $p_{\max } / p=10$ contains $20 \%$ of the area but more than $55 \%$ of the flux, while the region outside $p_{\max } / p=100$ contains contains less than $5 \%$ of the area but $20 \%$ of the flux.

We also use $p_{\max } / p$ from the $1 \mathrm{~km}$ simulation to compare the vertical advective fluxes across resolutions and also against the coarse-grained fields from the $1 \mathrm{~km}$ simulation (Figure 13a,b), comparing the role played by the vorticity-strain values that are resolvable at the lower resolutions and the additional contributions coming from the values that are not resolved. The advective flux first increases and then rapidly decreases as $p_{\max } / p$ increases, indicating a net downward flux that results from large cancellations between upward and downward fluxes in different regions. The upward flux at lower $p_{\max } / p$ is a result of the strong upwelling in the AVD regions which is present closer to the peak of the JPDF, and is much stronger at $1 \mathrm{~km}$ resolution than at lower resolutions (Figure 11). When considering only the eddy advective flux, we do not see this upwelling signal at smaller $p_{\max } / p$, which is consistent with Figures $9 \mathrm{~b}$ and $10 \mathrm{~b}$.

The difference between the simulations versus the coarsened fields is not very dramatic. This analysis helps re-emphasize the role played by smaller scales, which are unresolvable on coarser grids and occupy a very small fraction of the surface area, in fluxing tracer to depth. At the lower resolutions, changing from $20 \mathrm{~km}$ to $5 \mathrm{~km}$, the additional flux is a result of simply resolving a wider range of vorticity-strain values. At $1 \mathrm{~km}$ resolution, the flux even at the lower vorticitystrain values is modified, which is likely due to strengthening of fronts and the the dynamics of the fronts changing from being QG-like to SG-like as resolution increases.

\section{Discussion}

Here we have demonstrated that surface vorticity-strain JPDFs are a powerful diagnostic tool that can easily distinguish between different flow regions, and help study the impact of these by 
providing a convenient frame to perform conditional averages. We showed that the JPDF has a distinct shape, which has been noted previously in observations (eg. Shcherbina et al. 2013; Berta et al. 2020) and models (eg. Rocha et al. 2016), and appears to be shaped in part by flow instabilities. Conditioning vertical velocities and vertical advective tracer fluxes on strain and vorticity helped highlight the outsized impact played by smaller-scale flow features, particularly in the strain dominated (SD) regions, in the vertical exchange of a tracer across the base of the mixed layer: $\sim 20 \%$ of the vertical flux is achieved in fronts that occupy less than $\sim 5 \%$ of the surface area.

This study has helped address an obvious question that has arisen from observational campaigns centered around individual fronts (Shcherbina et al. 2013; Mahadevan et al. 2020) — even though fronts are observed to be sites of significantly enhanced transport, are they widespread enough to play an important role in setting the large scale tracer budgets? We have shown here that submesoscale SD regions and fronts do end up playing an important role on the net transport. As horizontal resolution is increased, we find that the tracer fluxes increase not only as a result of additional flux at smaller scales being resolved, but also due to the contrubution from the largescales increasing in response to the resolved small-scale flows. Therefore more emphasis needs to be placed on their parameterization, particularly their role in exchange between the mixed layer and the interior (Fox-Kemper et al. 2008; Uchida et al. 2020; Bachman and Klocker 2020).

One of the caveats of our study is that we condition the flux at depth on the surface properties. It is possible that some features at depth may not be directly related to the surface vorticity-strain, but rather to only the part of the surface horizontal flows that have not decayed at that level; generally smaller features decay more rapidly with depth than larger features. More analysis is needed to assess how important this effect is, and it will be part of future work. A counter-argument is that it is important to condition on surface properties, because that is the region that interacts with the 
atmosphere and supplies tracers to depth (or allows for outgassing of tracers leaving the ocean). So even if a number of small fronts decay and merge to form a single weaker front at depth, the transport in this deeper front would depend critically on how much tracer reaches it via the smaller fronts.

Our highest resolution simulations are at $1 \mathrm{~km}$, which is sufficient to resolve the interior baroclinic instability, the fronts that form at the surface due to the associated mesoscale eddies, and to some degree even the mixed layer instabilities (Balwada et al. 2018; Uchida et al. 2019). We likely do not resolve the full impact of smaller submesoscale dynamics or instabilities, e.g. symmetric instability, which are suggested to enhance vertical transport across the mixed layer even further (Brannigan 2016; Smith et al. 2016). Regardless, it is very likely that further resolving the submesoscale will further enhance the tracer flux across the base of the mixed layer, via the formation of powerful small-scale fronts, even if the mixed layers become shallower due to enhanced restratification (Balwada et al. 2018).

The channel simulations considered here are representative of the Antarctic Circumpolar Current, and maybe to a lesser degree the separated western boundary currents. These are regions where deep isopycnals shoal to the surface, and the large-scale hydrography is conducive to exchanging tracers between the surface and deep ocean. The role of these regions in being important sites for exchange across the mixed layer has long been known, as inferred from tracer distributions (Stommel 1979; Williams et al. 1995; Sallée et al. 2010; Marshall and Speer 2012). Our study speaks to the role of submesoscales in tracer dynamics of these regions, particularly in the winter when a strong density jump across the base of the mixed layer is not present. The impact of submesoscales in regions where isopycnals are relatively flat, and an adiabatic surface-interior pathway is absent, is still relatively unknown and likely to be weak. 
Our work has shown that statistical relationships between the surface kinematic properties and vertical exchange at depth exist. This suggests that the next-generation of satellite-based surface flow estimates, e.g. from SWOT (Morrow et al. 2019) or DopplerScatts (Rodríguez et al. 2018), can potentially help inform how climatically important tracers are being fluxed vertically and stored in the ocean. Some efforts in establishing dynamics based methods to reconstruct maps of vertical velocities are already underway (e.g. Qiu et al. 2020), and we suggest that statistical or machine learning approaches that directly infer the net fluxes will also be immensely fruitful.

\section{APPENDIX A}

\section{Tracer gradient kinematics}

Here we review of the fundamentals of the local kinematics of stirring in two dimensions; see Okubo (1970), Weiss (1991), Lapeyre et al. (1999), or Majda (2003) for background.

The advection of a two-dimensional tracer $c=c(x, y, t)$ is described by

$$
\frac{\mathrm{d} c}{\mathrm{~d} t} \doteq \partial_{t} c+\boldsymbol{u} \cdot \nabla c=0,
$$

where the velocity $\boldsymbol{u}=(u, v)$ may be divergent. Taking the gradient of (A1) gives the vector equation for the evolution of the gradient,

$$
\frac{\mathrm{d} \nabla c}{\mathrm{~d} t}=-\Lambda \nabla c, \quad \text { where } \quad \Lambda=\left[\begin{array}{ll}
u_{x} & v_{x} \\
u_{y} & v_{y}
\end{array}\right]
$$

is the transpose velocity gradient tensor, and $\nabla c$ is taken to be a column vector. In the "frozenfield" limit, where the velocity field is assumed to evolve slowly relative to the evolution of the tracer gradient, $\Lambda$ is taken to be constant, and (A2) describes a dynamical system for $\nabla c$ in the Lagrangian frame. 
The transpose velocity gradient tensor can also be expressed as

$$
\Lambda=\frac{1}{2}\left[\begin{array}{ll}
\Delta+\sigma_{n} & \sigma_{s}+\zeta \\
\sigma_{s}-\zeta & \Delta-\sigma_{n}
\end{array}\right]
$$

where the definitions in (1) are used. The eigenvalues of $\Lambda$ are

$$
\lambda_{ \pm}=\frac{1}{2}(\Delta \pm \sqrt{\Omega}), \quad \text { where } \quad \Omega=\sigma^{2}-\zeta^{2}
$$

is the Okubo-Weiss parameter (Okubo 1970; Weiss 1991). As long as the eigenvalues are distinct, they have linearly-independent eigenvectors $\boldsymbol{v}_{ \pm}$, and one can express the tracer gradient as a linear combination of the eigenvectors, giving the full solution

$$
\nabla c=a_{+}(0) e^{-\lambda_{+} t} \boldsymbol{v}_{+}+a_{-}(0) e^{-\lambda_{-} t} \boldsymbol{v}_{-},
$$

where $a_{ \pm}(0)$ are determined by the initial conditions ${ }^{2}$.

In the limit of non-divergent flow, with $\Delta=0$, the eigenvalues are purely real if $\Omega>0$ (straindominant), or purely imaginary if $\Omega<0$ (vorticity-dominant). In the former strain-dominant case, the eigenvalues are equal and opposite, leading the gradient to increase in the $\boldsymbol{v}_{-}$direction and decrease in the $\boldsymbol{v}_{+}$direction. In the vorticity-dominant case, the gradient simply rotates without changing its magnitude.

Divergent flow will change the magnitude of the gradient regardless of the sign of $\Omega$, decreasing gradients for $\Delta>0$ and increasing gradients for $\Delta<0$. The most relevant case occurs for convergent $(\Delta<0)$ strain-dominant $(\Omega>0)$ flow, where the convergence amplifies frontogenesis tendencies with growth rate $-\lambda_{-}=\Delta+\sqrt{\Omega}$. For sufficiently negative divergence, $-\lambda_{+}$will also be positive, and the gradient will contract in both directions.

It is also instructive to compute the evolution equation for the squared gradient. The transpose velocity gradient tensor may be decomposed into symmetric and antisymmetric parts, $\Lambda=S+A$,

\footnotetext{
${ }^{2}$ When $\lambda_{ \pm}$is complex, the solution can be expressed in terms of sin and cos with real coefficients and eigenvectors.
} 
where $S=\left(\Lambda+\Lambda^{\mathrm{T}}\right) / 2$ and $A=\left(\Lambda-\Lambda^{\mathrm{T}}\right) / 2$. The symmetric part may be orthogonally diagonalized as $\mathrm{S}=\mathrm{VDV}^{\mathrm{T}}$, where $\mathrm{V}^{\mathrm{T}}=\mathrm{V}^{-1}$ and $\mathrm{D}$ is a diagonal matrix with the eigenvalues of $\mathrm{S}$, which are $\delta \pm \sigma$, as its diagonal elements. Then $\mathrm{d} \nabla c / \mathrm{d} t=-\left(\mathrm{A}+\mathrm{VDV}^{\mathrm{T}}\right) \nabla c$. Multiplying by $\nabla c^{\mathrm{T}}$ and noting $\boldsymbol{v}^{\mathrm{T}} \mathrm{A} \boldsymbol{v}=0$ for any column vector $\boldsymbol{v}$, we find

$$
\frac{\mathrm{d}}{\mathrm{d} t} \frac{|\nabla c|^{2}}{2}=-\frac{\Delta+\sigma}{2} c_{\tilde{x}}^{2}-\frac{\Delta-\sigma}{2} c_{\tilde{y}}^{2}
$$

where $[\tilde{x} \tilde{y}]^{T}=\tilde{\boldsymbol{x}}=\mathrm{V}^{\mathrm{T}} \boldsymbol{x}$ are the coordinates with respect to the eigenbasis $\mathrm{V}$. Thus the vorticity has no effect on the tracer gradient magnitude, the strain strengthens gradients in one direction and decreases them in the other, and convergence uniformly strengthens gradients.

\section{APPENDIX B}

\section{Joint distributions and conditional means}

Consider a scalar field $F(x, y)$, along with the vorticity $\zeta(x, y)$ and the strain $\sigma(x, y)$, all defined on a control area $A$ (the domain) at some $z$ and $t$ (for clarity we suppress these arguments below). Then the quantity

$$
\tilde{F}(\zeta, \sigma) \doteq \iint_{A} F(x, y) \delta\left[\zeta^{\prime}(x, y)-\zeta\right] \delta\left[\sigma^{\prime}(x, y)-\sigma\right] \mathrm{d} x \mathrm{~d} y
$$

is the distribution of $F$ conditioned on strain and vorticity. Examples of distributions in terms of nonmonotonic variables include, in the atmospheric literature, binning transport in terms of moist potential temperature (Pauluis et al. 2008; Laliberté et al. 2015), and in oceanography, expressing transport in terms of temperature and salinity (Zika et al. 2012).

The spatial area integral of $F$ and the integral over vorticity-strain space of $\tilde{F}$ have to be equal,

$$
\iint_{A} F(x, y) \mathrm{d} x \mathrm{~d} y=\iint_{R} \tilde{F}\left(\zeta^{\prime}, \sigma^{\prime}\right) \mathrm{d} \zeta^{\prime} \mathrm{d} \sigma^{\prime}
$$

where $R$ is the range of vorticity and strain values found in spatial area $A$. Notice that if $F=1$, and $\tilde{F}$ is defined on finite-difference grids, then $\tilde{F}(\zeta, \sigma)$ is the number of points in $A$ with $\sigma^{\prime} \in$ 
$[\sigma, \sigma+\Delta \sigma)$ and $\zeta^{\prime} \in[\zeta, \zeta+\Delta \zeta)$, divided by $\Delta \sigma \Delta \zeta$. Thus the total spatial area covered by points with strain and vorticity in this range is $\tilde{F}(\zeta, \sigma) \Delta \sigma \Delta \zeta \Delta x \Delta y$. The joint probability distribution function (JPDF) is correspondingly defined as,

$$
P(\zeta, \sigma)=\frac{\tilde{F}(\zeta, \sigma) \Delta \sigma \Delta \zeta \Delta x \Delta y}{A} .
$$

The spatial mean, is defined as,

$$
\bar{F}=\frac{\iint_{A} F(x, y) \mathrm{d} x \mathrm{~d} y}{A},
$$

while the conditional mean of $F$, always conditioned on surface vorticity and strain in this study, is defined as

$$
\bar{F}^{\zeta \sigma}(\zeta, \sigma)=\frac{\iint F(x, y) \delta\left[\sigma^{\prime}(x, y)-\sigma\right] \delta\left[\zeta^{\prime}(x, y)-\zeta\right] \mathrm{d} x \mathrm{~d} y}{\iint \delta\left[\sigma^{\prime}(x, y)-\sigma\right] \delta\left[\zeta^{\prime}(x, y)-\zeta\right] \mathrm{d} x \mathrm{~d} y}
$$

Note the difference in notation between the spatial and conditional means.

\section{APPENDIX C}

\section{Potential for instabilities in vorticity-strain space}

Some facets of the distinctive shape of the JPDF can be understood as an equilibrium between the large-scale flow and instabilities forcing the generation of gradients, which cascade to smaller scales and leading to an expansion of the JPDF, and the smaller-scale instabilities and dissipation curbing the expansion, by limiting the strength of the gradients (McWilliams 2016; Bodner et al. 2019). While a complete theory for the shape is beyond the scope of this work, here we highlight how flows in different regions of the JPDF might be susceptible to different types of instabilities.

First we consider the kinematics of the flow, which allows us to identify regions in vorticitystrain space where tracer gradients will undergo rapid exponential growth. In the Lagrangian frame, tracer gradients evolve like $\exp \left(-\lambda_{ \pm} t\right)$, where $\lambda_{ \pm}=\frac{1}{2}(\Delta \pm \sqrt{\Omega})$ are the eigenvalues of the 
strain matrix, and $\Omega=\sigma^{2}-\zeta^{2}$ is the Okubo-Weiss parameter (see Appendix A). In the absence of divergence, regions with $\Omega>0$ (corresponding to the SD regions) will correspond to exponential growth of tracer gradients, with growth rate $-\lambda_{-}$; this rate is enhanced further in the presence of convergence $(\Delta<0)$. Figure Cla shows that the conditional mean of the normalized growth rate $\left(\overline{-\lambda}_{-}^{\zeta \sigma} /\left|f_{0}\right|\right)$ increases rapidly with increasing strain magnitude, in regions of $\Omega>0$. The corresponding spatial regions of very rapidly increasing tracer gradients, particularly for active tracers like buoyancy, are associated with very fast flows and can result in secondary instabilities (e.g. a particular instability that appears in these conditions is the ageostrophic anticyclonic instability, AAI, McWilliams 2016; Bachman and Klocker 2020).

The strength of the buoyancy gradients (Figure C1b) does not exactly follow the eigenvalues and is generally larger in regions of positive vorticity and large strain; as there are other factors apart from the growth rate that will determine how strong the gradients are.

The asymmetry of the JPDF along the vorticity axis can be understood by considering instabilities that depend on the sign of the Ertel PV, $q=(\boldsymbol{\omega}+f \hat{\boldsymbol{z}}) \cdot \nabla b ; \boldsymbol{\omega}=\nabla \times \boldsymbol{v}$ is the vorticity vector, $f$ is the Coriolis frequency, $b$ is the buoyancy. In the absence of flow variations along the direction of the flow, the flow is unstable to either inertial instability or symmetric instability if $f q<0$ (Hoskins 1974). Alternatively, the flow is unstable when the nondimensionalized Ertel PV is less than 0 , i.e.

$$
\Pi=\frac{q}{f N^{2}}=1+R o-R i^{-1}<0,
$$

where geostrophic balance and thermal wind is assumed to hold at leading order; $R o=\zeta / f$, $R i=N^{2} /\left|\partial_{z} \mathbf{u}\right|^{2}, N^{2}=\bar{b}_{z}$, and $\partial_{z} \mathbf{u}$ is the vertical shear. A detailed summary of the different classes of instabilities that arise is given in Thomas et al. (2013). For $R i \gg 1$, the flow is subject to inertial instability if $R o<-1$, which suggests that the JPDF should be limited on the anticyclonic side to values with $\zeta / f \geq-1$, since the regions with higher values will be unstable and rapidly 
deform towards more stable flow orientations. For sufficiently small $R i$, such that $R i^{-1}>1+R o$, symmetric instability is possible on the cyclonic side as well.

Recently Buckingham et al. (2021a,b) developed a general stability criterion that applies to flow with curvature. A key result is that, for small enough $R i$, cyclonic flows should be more unstable than anticyclonic flows. The criterion for instability is analogous to the Hoskins criterion with $f$ replaced by the absolute angular momentum $L$, or $L q<0$. The nondimensional form of the criterion, analogous to $(\mathrm{C} 1)$, is

$$
\Phi=(1+C u)(1+R o)-(1+C u)^{2} R i-1<0,
$$

where $C u=2 V /(f R)$ is the curvature number, with $V$ being the geostrophic speed and $R$ being the radius of curvature. Here we estimated the radius of curvature as (Theisel 1995)

$$
R=\frac{\left(u^{2}+v^{2}\right)^{3 / 2}}{u^{2} v_{x}-v^{2} u_{y}+u v\left(v_{y}-u_{x}\right)} .
$$

Figure $\mathrm{C} 1 \mathrm{c}$ and d show the conditional means of the instability criteria, $\bar{\Pi}^{\zeta \sigma}$ and $\bar{\Phi}^{\zeta \sigma}$. Note that unlike panel a, which is an estimate of a growth rate, panels $\mathrm{c}$ and $\mathrm{d}$ are regime diagrams, indicating instability where values are negative. Both panels show inertial instability on the anticyclonic side, and symmetric instability in the SD region $(\Omega>0)$. Interestingly, the criterion that accounts for curvature, $\Phi$, is on an average negative on the cyclonic side as well in the SD region, and shows a degree of stabilization for the AVD regions. The CVD regions with the strongest vorticity, which are likely associated with strong cyclones, remain stable under all criteria, and their strength is likely associated with the model resolution.

Acknowledgments. DB and ARG acknowledge support from the NSF grant OCE-1756882. KSS, RPA and QX acknowledge support from NASA award 80NSSC20K1142. This work would not have been possible without the tools provided by and maintained by the Pangeo community (https://pangeo.io/). The code for the analysis presented here is available at https: 
//github.com/dhruvbalwada/vorticity-strain-conditioning, and most of the necessary data is at https://catalog.pangeo.io/browse/master/ocean/channel/, which allows the analysis to be done directly on the cloud (Abernathey et al. 2020).

\section{References}

Abernathey, R., and G. Haller, 2018: Transport by Lagrangian vortices in the Eastern Pacific. Journal of Physical Oceanography, 48 (3), 667-685.

Abernathey, R., and Coauthors, 2020: Cloud-native repositories for big scientific data. Authorea Preprints.

Adams, K. A., P. Hosegood, J. R. Taylor, J.-B. Sallée, S. Bachman, R. Torres, and M. Stamper, 2017: Frontal circulation and submesoscale variability during the formation of a Southern Ocean mesoscale eddy. Journal of Physical Oceanography, 47 (7), 1737-1753.

Archer, M., A. Schaeffer, S. Keating, M. Roughan, R. Holmes, and L. Siegelman, 2020: Observations of submesoscale variability and frontal subduction within the mesoscale eddy field of the Tasman Sea. Journal of Physical Oceanography, 50 (5), 1509-1529.

Armi, L., and P. Flament, 1985: Cautionary remarks on the spectral interpretation of turbulent flows. Journal of Geophysical Research: Oceans, 90 (C6), 11 779-11 782.

Bachman, S. D., and A. Klocker, 2020: Interaction of jets and submesoscale dynamics leads to rapid ocean ventilation. Journal of Physical Oceanography, 50 (10), 2873-2883.

Balwada, D., K. S. Smith, and R. Abernathey, 2018: Submesoscale vertical velocities enhance tracer subduction in an idealized Antarctic Circumpolar Current. Geophysical Research Letters, 45 (18), 9790-9802. 
Barkan, R., M. J. Molemaker, K. Srinivasan, J. C. McWilliams, and E. A. D'Asaro, 2019: The role of horizontal divergence in submesoscale frontogenesis. Journal of Physical Oceanography, $49(6), 1593-1618$.

Berta, M., and Coauthors, 2020: Submesoscale kinematic properties in summer and winter surface flows in the northern Gulf of Mexico. Journal of Geophysical Research: Oceans, 125 (10), e2020JC016 085.

Boccaletti, G., R. Ferrari, and B. Fox-Kemper, 2007: Mixed layer instabilities and restratification. Journal of Physical Oceanography, 37 (9), 2228-2250.

Bodner, A. S., B. Fox-Kemper, L. P. Van Roekel, J. C. McWilliams, and P. P. Sullivan, 2019: A perturbation approach to understanding the effects of turbulence on frontogenesis. Journal of Fluid Mechanics, $\mathbf{8 8 3}$.

Brannigan, L., 2016: Intense submesoscale upwelling in anticyclonic eddies. Geophysical Research Letters, 43 (7), 3360-3369, doi:10.1002/2016GL067926.

Buckingham, C. E., J. Gula, and X. Carton, 2021a: The role of curvature in modifying frontal instabilities. Part I: Review of theory and presentation of a nondimensional instability criterion. J. Phys. Oceanogr., 51, 299-315.

Buckingham, C. E., J. Gula, and X. Carton, 2021b: The role of curvature in modifying frontal instabilities. Part II: Application of the criterion to curved density fronts at low Richardson numbers. J. Phys. Oceanogr., 51, 317-341.

Callies, J., R. Barkan, and A. N. Garabato, 2020: Time scales of submesoscale flow inferred from a mooring array. Journal of Physical Oceanography, 50 (4), 1065-1086. 
Callies, J., G. Flierl, R. Ferrari, and B. Fox-Kemper, 2016: The role of mixed-layer instabilities in submesoscale turbulence. Journal of Fluid Mechanics, 788, 5-41.

Capet, X., J. C. McWilliams, M. J. Molemaker, and A. Shchepetkin, 2008: Mesoscale to submesoscale transition in the California Current System. Part II: Frontal processes. J. Phys. Oceanogr., 38 (1), 44-64.

Ferrari, R., 2011: A frontal challenge for climate models. Science, 332 (6027), 316-317, doi: 10.1126/science. 1203632 .

Fox-Kemper, B., R. Ferrari, and R. Hallberg, 2008: Parameterization of mixed layer eddies. Part I: Theory and diagnosis. Journal of Physical Oceanography, 38 (6), 1145-1165.

Franks, P. J., 2005: Plankton patchiness, turbulent transport and spatial spectra. Marine Ecology Progress Series, 294, 295-309.

Freilich, M. A., and A. Mahadevan, 2019: Decomposition of vertical velocity for nutrient transport in the upper ocean. Journal of Physical Oceanography, 49 (6), 1561-1575.

Gula, J., M. J. Molemaker, and J. C. McWilliams, 2014: Submesoscale cold filaments in the Gulf Stream. Journal of Physical Oceanography, 44 (10), 2617-2643.

Haller, G., 2015: Lagrangian coherent structures. Annual Review of Fluid Mechanics, 47, 137162.

Ho, D. T., C. S. Law, M. J. Smith, P. Schlosser, M. Harvey, and P. Hill, 2006: Measurements of air-sea gas exchange at high wind speeds in the Southern Ocean: Implications for global parameterizations. Geophysical Research Letters, 33 (16).

Hoskins, B., 1974: The role of potential vorticity in symmetric stability and instability. Quarterly Journal of the Royal Meteorological Society, 100 (425), 480-482. 
Hoskins, B. J., and F. P. Bretherton, 1972: Atmospheric frontogenesis models: Mathematical formulation and solution. Journal of the Atmospheric Sciences, 29 (1), 11-37.

Klocker, A., 2018: Opening the window to the Southern Ocean: The role of jet dynamics. Science advances, 4 (10), eaao4719.

Laliberté, F., J. Zika, L. Mudryk, P. J. Kushner, J. Kjellsson, and K. Döös, 2015: Constrained work output of the moist atmospheric heat engine in a warming climate. Science, 347, 540-543.

Lapeyre, G., and P. Klein, 2006: Dynamics of the upper oceanic layers in terms of surface quasigeostrophy theory. Journal of Physical Oceanography, 36 (2), 165-176.

Lapeyre, G., P. Klein, and B. L. Hua, 1999: Does the tracer gradient vector align with the strain eigenvectors in 2d turbulence? Physics of Fluids, 11 (12), 3729-3737, doi:10.1063/1.870234.

Large, W. G., J. C. McWilliams, and S. C. Doney, 1994: Oceanic vertical mixing: A review and a model with a nonlocal boundary layer parameterization. Reviews of Geophysics, 32 (4), $363-403$.

Lévy, M., P. J. S. Franks, and K. S. Smith, 2018: The role of submesoscale currents in structuring marine ecosystems. Nature Communications, 9 (1), 4758, doi:10.1038/s41467-018-07059-3.

Lévy, M., P. Klein, and A.-M. Treguier, 2001: Impact of sub-mesoscale physics on production and subduction of phytoplankton in an oligotrophic regime. Journal of Marine Research, 59 (4), $535-565$.

Mahadevan, A., A. Pascual, D. L. Rudnick, S. Ruiz, J. Tintoré, and E. D’Asaro, 2020: Coherent pathways for vertical transport from the surface ocean to interior. Bulletin of the American Meteorological Society, 101 (11), E1996-E2004. 
Mahadevan, A., and A. Tandon, 2006: An analysis of mechanisms for submesoscale vertical motion at ocean fronts. Ocean Modelling, 14 (3-4), 241-256.

Majda, A. J., 2003: Introduction to PDEs and Waves for the Atmosphere and Ocean. American Mathematical Soc., 234 pp.

Marshall, D., 1997: Subduction of water masses in an eddying ocean. Journal of Marine Research, 55, 201-222.

Marshall, J., A. Adcroft, C. Hill, L. Perelman, and C. Heisey, 1997: A finite-volume, incompressible navier stokes model for studies of the ocean on parallel computers. Journal of Geophysical Research: Oceans, 102 (C3), 5753-5766, doi:https://doi.org/10.1029/ 96JC02775, URL https://agupubs.onlinelibrary.wiley.com/doi/abs/10.1029/96JC02775, https: //agupubs.onlinelibrary.wiley.com/doi/pdf/10.1029/96JC02775.

Marshall, J., and K. Speer, 2012: Closure of the meridional overturning circulation through Southern Ocean upwelling. Nature Geoscience, 5 (3), 171-180.

Marshall, J. C., R. G. Williams, and A. G. Nurser, 1993: Inferring the subduction rate and period over the North Atlantic. J. Phys. Oceanogr., 23 (7), 1315-1329.

McWilliams, J. C., 2016: Submesoscale currents in the ocean. Proceedings of the Royal Society A: Mathematical, Physical and Engineering Sciences, 472 (2189), 20160117.

Morrow, R., and Coauthors, 2019: Global observations of fine-scale ocean surface topography with the Surface Water and Ocean Topography (SWOT) mission. Frontiers in Marine Science, 6, 232, doi:10.3389/fmars.2019.00232.

Okubo, A., 1970: Horizontal dispersion of floatable particles in the vicinity of velocity singularities such as convergences. Deep-Sea Res., 17, 445-454. 
Olita, A., and Coauthors, 2017: Frontal dynamics boost primary production in the summer stratified Mediterranean Sea. Ocean Dynamics, 67 (6), 767-782.

Omand, M. M., E. A. DAsaro, C. M. Lee, M. J. Perry, N. Briggs, I. Cetinić, and A. Mahadevan, 2015: Eddy-driven subduction exports particulate organic carbon from the spring bloom. Science, 348 (6231), 222-225, doi:10.1126/science. 1260062.

Pauluis, O., A. Czaja, and R. Korty, 2008: The global atmospheric circulation on moist isentropes. Science, 321, 1075-1078.

Price, J. F., R. A. Weller, and R. R. Schudlich, 1987: Wind-driven ocean currents and Ekman transport. Science, 238 (4833), 1534-1538.

Qiu, B., S. Chen, P. Klein, H. Torres, J. Wang, L.-L. Fu, and D. Menemenlis, 2020: Reconstructing upper-ocean vertical velocity field from sea surface height in the presence of unbalanced motion. Journal of Physical Oceanography, 50 (1), 55-79.

Ramachandran, S., A. Tandon, and A. Mahadevan, 2014: Enhancement in vertical fluxes at a front by mesoscale-submesoscale coupling. Journal of Geophysical Research: Oceans, 119 (12), $8495-8511$.

Rocha, C. B., S. T. Gille, T. K. Chereskin, and D. Menemenlis, 2016: Seasonality of submesoscale dynamics in the Kuroshio Extension. Geophysical Research Letters, 43 (21), 11-304.

Rodríguez, E., A. Wineteer, D. Perkovic-Martin, T. Gál, B. W. Stiles, N. Niamsuwan, and R. Rodriguez Monje, 2018: Estimating ocean vector winds and currents using a Ka-band pencil-beam Doppler scatterometer. Remote Sensing, 10 (4), 576. 
Roullet, G., J. C. Mcwilliams, X. Capet, and M. J. Molemaker, 2012: Properties of steady geostrophic turbulence with isopycnal outcropping. Journal of Physical Oceanography, 42 (1), $18-38$.

Ruiz, S., and Coauthors, 2019: Effects of oceanic mesoscale and submesoscale frontal processes on the vertical transport of phytoplankton. Journal of Geophysical Research: Oceans, 124 (8), $5999-6014$.

Sallée, J.-B., K. Speer, S. Rintoul, and S. Wijffels, 2010: Southern Ocean thermocline ventilation. J. Phys. Oceanogr., 40 (3), 509-529.

Shakespeare, C., 2015: On the generation of waves during frontogenesis. Ph.D. thesis, University of Cambridge.

Shcherbina, A. Y., E. A. D’Asaro, C. M. Lee, J. M. Klymak, M. J. Molemaker, and J. C. McWilliams, 2013: Statistics of vertical vorticity, divergence, and strain in a developed submesoscale turbulence field. Geophysical Research Letters, 40 (17), 4706-4711.

Siegelman, L., P. Klein, P. Rivière, A. F. Thompson, H. S. Torres, M. Flexas, and D. Menemenlis, 2020: Enhanced upward heat transport at deep submesoscale ocean fronts. Nature Geoscience, $13(1), 50-55$.

Sinha, A., D. Balwada, N. Tarshish, and R. Abernathey, 2019: Modulation of lateral transport by submesoscale flows and inertia-gravity waves. Journal of Advances in Modeling Earth Systems, $11(4), 1039-1065$.

Smith, K. M., P. E. Hamlington, and B. Fox-Kemper, 2016: Effects of submesoscale turbulence on ocean tracers. Journal of Geophysical Research: Oceans, 121 (1), 908-933. 
Stommel, H., 1979: Determination of water mass properties of water pumped down from the Ekman layer to the geostrophic flow below. Proceedings of the National Academy of Sciences, 76 (7), 3051-3055.

Su, Z., J. Wang, P. Klein, A. F. Thompson, and D. Menemenlis, 2018: Ocean submesoscales as a key component of the global heat budget. Nature communications, 9 (1), 1-8.

Theisel, H., 1995: Vector field curvature and applications. Ph.D. thesis, Verlag nicht ermittelbar.

Thomas, L. N., A. Tandon, and A. Mahadevan, 2008: Submesoscale processes and dynamics. Ocean modeling in an Eddying Regime, 177, 17-38.

Thomas, L. N., J. R. Taylor, R. Ferrari, and T. M. Joyce, 2013: Symmetric instability in the Gulf Stream. Deep Sea Research Part II: Topical Studies in Oceanography, 91, 96-110.

Uchida, T., D. Balwada, R. Abernathey, G. McKinley, S. Smith, and M. Lévy, 2019: The contribution of submesoscale over mesoscale eddy iron transport in the open Southern Ocean. Journal of Advances in Modeling Earth Systems, 11 (12), 3934-3958.

Uchida, T., D. Balwada, R. P. Abernathey, G. A. McKinley, S. K. Smith, and M. Lévy, 2020: Vertical eddy iron fluxes support primary production in the open Southern Ocean. Nature Communications, 11 (1), 1-8.

Weiss, J., 1991: The dynamics of enstrophy transfer in two-dimensional hydrodynamics. Physica $D, 48,273-294$.

Williams, R. G., J. C. Marshall, and M. A. Spall, 1995: Does Stommel's mixed layer "demon" work? J. Phys. Oceanogr., 25 (12), 3089-3102. 

nates. J. Phys. Oceanogr., 42, 708-724. doi:10.3390/fluids5010002.

Zhang, W., C. L. Wolfe, and R. Abernathey, 2020: Role of surface-layer coherent eddies in potential vorticity transport in quasigeostrophic turbulence driven by eastward shear. Fluids, $\mathbf{5}$ (2),

Zika, J. D., M. H. England, and W. P. Sijp, 2012: The ocean circulation in thermohaline coordi- 


\section{LIST OF FIGURES}

Fig. 1. The surface vorticity (a), tracer concentration (b), vertical velocity (c), and vertical tracer flux (d) at 100m depth 10 days after the tracer source is introduced at the surface in a small region upstream of the ridge. (e) Histogram of vertical flux in the chosen region at 3 different times. (f) Time series of the mean tracer flux in the chosen region. Notice that the mean tracer flux is almost two orders of magnitude smaller than the range of the instantaneous fluxes.

Fig. 2. Snapshot of surface vorticity $(a, d, g)$, surface strain (b,e,h), tracer concentration at base of mixed layer (c,f,i) at $1 \mathrm{~km}$ (top row), $5 \mathrm{~km}$ (middle row) and $20 \mathrm{~km}$ (bottom row) resolutions. The vorticity and strain are normalized by the Coriolis frequency. The snapshot are taken 4 months after the tracer forcing is turned on. The horizontal dashed lines at $y=500$ and $1500 \mathrm{~km}$ in the upper left figure encompass the analysis region used for most of the diagnostics in this study, and the dashed box downstream of the ridge indicates the region that is used for the fields in Figure 4.

Fig. 3. Surface vorticity-strain JPDF for the $1 \mathrm{~km}$ simulation. The gray contour lines correspond to the outer limits $\left(P(\zeta, \sigma)=10^{-5}\right)$ for the JPDFs from the $1 \mathrm{~km}, 5 \mathrm{~km}$ and $20 \mathrm{~km}$ simulations, with the innermost contour corresponding to the lowest resolution and the outermost contour corresponding to the highest resolution. In this and all following plots on the $\zeta / f_{0}$ vs $\sigma /\left|f_{0}\right|$ plane, the dashed lines are the $\sigma=|\zeta|$ lines. These lines demarcate the boundaries between the strain dominated (SD), anticyclonic vorticity dominated (AVD), and cyclonic vorticity dominated (CVD) regions.

Fig. 4. Surface vorticity-strain based flow decomposition. Surface vorticity (a) and surface strain (c) in a large scale anticyclonic meander downstream (dashed box in Figure 2). (b) The JPDF corresponding to this region. Bottom row shows the surface vorticity decomposed based on where the grid points lie in the JPDF; corresponding to the AVD (d), CVD (e), and $\mathrm{SD}$ (f) regions.

Fig. 5. Properties of a typical front. The surface temperature (a), surface vorticity (b), surface strain (c), and the vorticity-strain JPDF (d) in a region with a strong front. A depth-across front section of the temperature (e), vertical velocity (f), and tracer on day $8(\mathrm{~g})$ and $10(\mathrm{~h})$ after the tracer forcing is turned on. The black contours in $(\mathrm{a}, \mathrm{e}, \mathrm{f}, \mathrm{g}, \mathrm{h})$ are some chosen temperature contours to highlight the front. The yellow contours in (e) show the meridional velocity, which is northwards, decaying away from the front.

Fig. 6. Relationship of surface divergence to vorticity and strain. (top panel) The mean surface divergence conditioned on surface vorticity-strain. (bottom two rows) Slices of the 3D vorticity-strain-divergence JPDF at particular values of surface divergence (as indicated in panel titles); top row corresponds to convergent regions and bottom row to divergent regions. The gray contours and dashed lines are the same as in Figure 3.

Fig. 7. Surface vorticity-strain JPDFs for the $5 \mathrm{~km}$ (a) and $20 \mathrm{~km}$ (b) simulation, and for the coarsegrained velocities from the $1 \mathrm{~km}$ simulation to $5 \mathrm{~km}$ (c) and $20 \mathrm{~km}$ (d) respectively. The gray contours and dashed lines are the same as in Figure 3. Note the changing axis limits with resolution, and relative to Figure 3.

Fig. 8. Conditional mean of the vertical velocity conditioned on the surface vorticity and strain at different resolutions (columns) and depths (rows). The top row is for $1 \mathrm{~km}$ resolution, followed by the $5 \mathrm{~km}$ and then the $20 \mathrm{~km}$. The first column corresponds to the middle of the mixed layer ( $50 \mathrm{~m}$ for $1 \mathrm{~km}, 75 \mathrm{~m}$ for $5 \mathrm{~km}$ and $90 \mathrm{~m}$ for $20 \mathrm{~km}$ ), the second column to 
the base of the mixed layer $(100 \mathrm{~m}$ for $1 \mathrm{~km}, 150 \mathrm{~m}$ for $5 \mathrm{~km}$ and $180 \mathrm{~m}$ for $20 \mathrm{~km}$ ), and the third column to a fixed depth of $250 \mathrm{~m}$. The gray contours and dashed lines are the same as in Figure 3. Note the changing axis limits with resolution.

Fig. 9. Conditional mean of different components of the tracer flux conditioned on the surface vorticity and strain; the components being (a) the total advective flux, (b) the eddy advective flux, (c) the surface flux, and (d) the diffusive flux. Notice that the different panels are for different depths and have different color ranges, the color ranges were chosen to allow a comparison across different regions of the JPDF rather than across figure panels. The diffusive flux is computed at the depth of $50 \mathrm{~m}$, which is the middle of the mixed layer - where the parameterized KPP boundary layer diffusivity is the highest (not shown), and the advective fluxes are computed at the depth of $100 \mathrm{~m}$, which is the base of the mixed layer. The gray contours and dashed lines are the same as in Figure 3.

Fig. 10. The contribution of regions corresponding to different parts of the surface vorticity-strain JPDF to tracer transport for the different components of the flux - (a) total advective flux, (b) eddy advective flux, (c) surface flux, and (d) diffusive flux. The gray contours and dashed lines are the same as in Figure 3.

Fig. 11. Vertical structure of different tracer flux components in the $1 \mathrm{~km}$ (top row) and $5 \mathrm{~km}$ (bottom row) resolution simulations, separated into components based on the regions in the JPDF. The first column $(\mathrm{a}, \mathrm{d})$ shows the total advective flux $(\overline{w C})$; the second column $(\mathrm{b}, \mathrm{e})$ shows the diffusive flux and the surface flux (inverted red triangles); and the third column (c, f) shows the eddy advective flux $\left(\overline{w^{\prime} C^{\prime}}\right)$ integrated over the parts of the JPDF corresponding to $\mathrm{SD}, \mathrm{AVD}$ and CVD region. The sum of the parts is shown as the dashed red line. For the $1 \mathrm{~km}$ simulation we have divided contribution from the SD region into $\mathrm{SD}^{>5 \mathrm{~km}}$ and $\mathrm{SD}^{<5 \mathrm{~km}}$ regions respectively. .

Fig. 12. Different flux components integrated outward from the maximum of the JPDF $\left(p_{\max }\right)$ is present to contours of decreasing probabilities $(p)$ in the surface vorticity-strain JPDF. The integral is plotted as a function of $p_{\max } / p$, where $p_{\max }$ is the probability at the maximum of the JPDF. As shown in section $2 \mathrm{c}$, higher values of $p_{\max } / p$ generally correspond to smallerscale features, and thus the $\mathrm{x}$-axis in this plot serves as a rough proxy for scales. Each curve asymptotes to the respective total flux at the corresponding depth. (a) The eddy advective flux at $100 \mathrm{~m}$, surface flux, and diffusive fluxes at $50 \mathrm{~m}$ for the $1 \mathrm{~km}$ simulation. (b) The flux fraction, defined as the integrated flux divided by the total flux, for the different components shown in (a). The dotted black line (axis shown on right) corresponds to the spatial area fraction contained in the region corresponding to $p_{\max } / p$ for the $1 \mathrm{~km}$ simulation.

Fig. 13. The total advective flux (a) and the eddy advective flux (b) at the base of the mixed layer for different resolutions and also for different coarsening scales applied to the $1 \mathrm{~km}$ simulation. The $\mathrm{x}$-axis is the same as in Figure 12. Black markers at the bottom of (a) indicate roughly where the outer-most probability contours of $20 \mathrm{~km}$ and $5 \mathrm{~km}$ simulations lie relative to the $1 \mathrm{~km}$ JPDF.

Fig. C1. Different criterion for instabilities and the strength of buoyancy gradients conditioned on vorticity-strain. The conditional means of (a) the normalized eigenvalue corresponding to rate of exponential growth of tracer gradients, (b) the absolute value of the buoyancy gradients, (c) the non-dimensionalized Ertel PV (П, equation C1), and (d) the generalized stability criterion ( $\Phi$, equation C2) from Buckingham et al. (2021a). Negative values of $\Pi$ or $\Phi$ suggest potential for instability. The gray contours and dashed lines are the same as in Figure 3. 

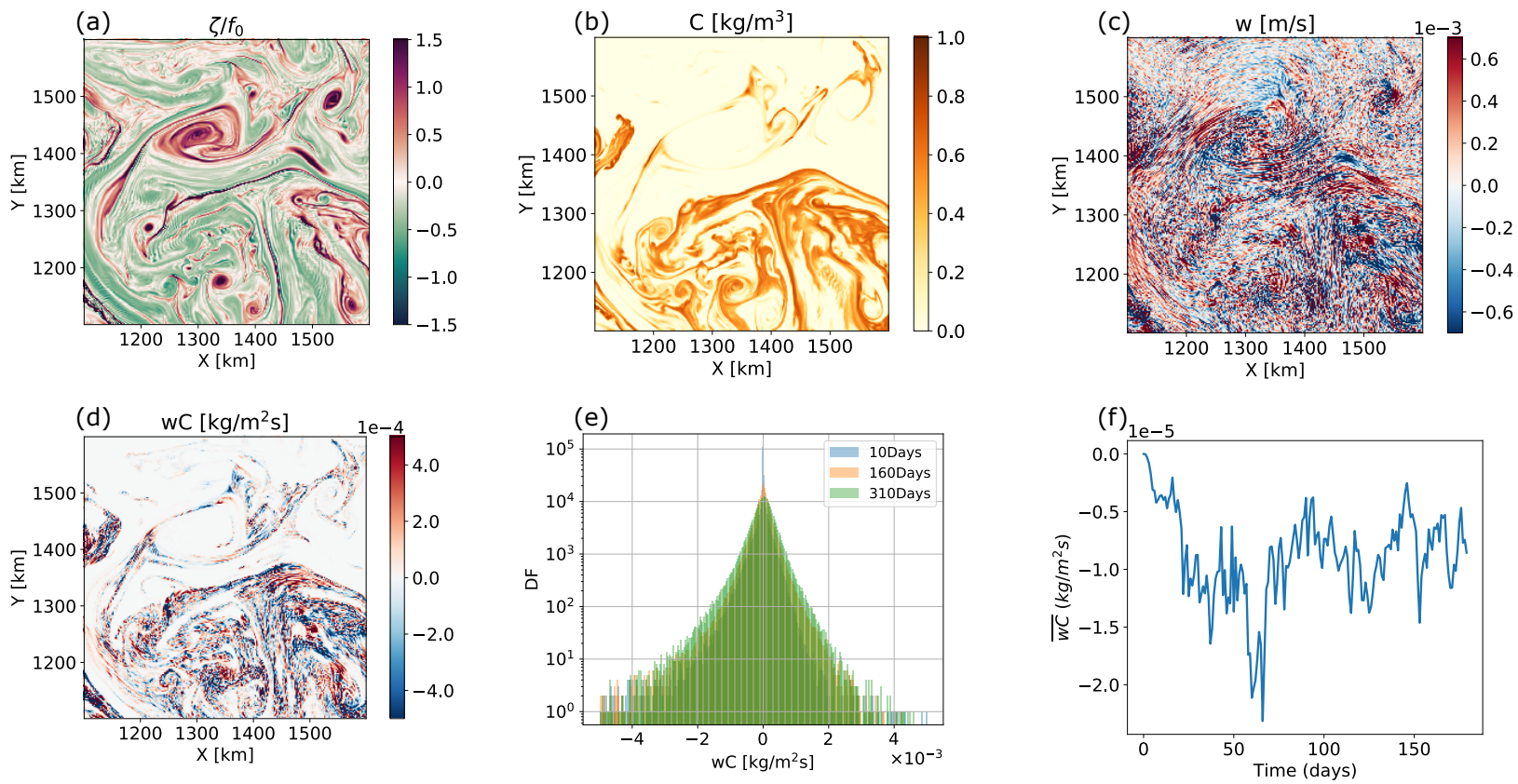

FIG. 1. The surface vorticity (a), tracer concentration (b), vertical velocity (c), and vertical tracer flux (d) at (e) Histogram of vertical flux in the chosen region at 3 different times. (f) Time series of the mean tracer flux in the chosen region. Notice that the mean tracer flux is almost two orders of magnitude smaller than the range of the instantaneous fluxes. 


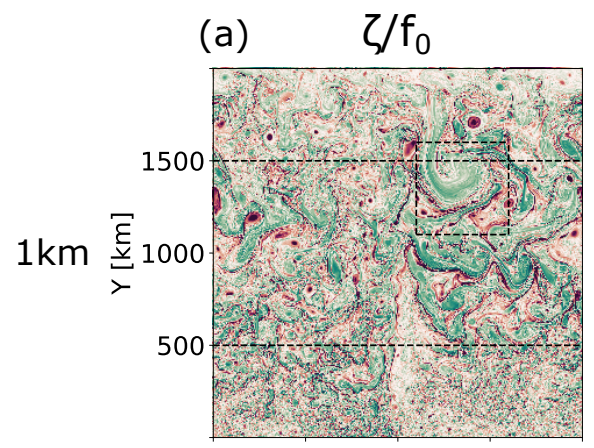

(d)

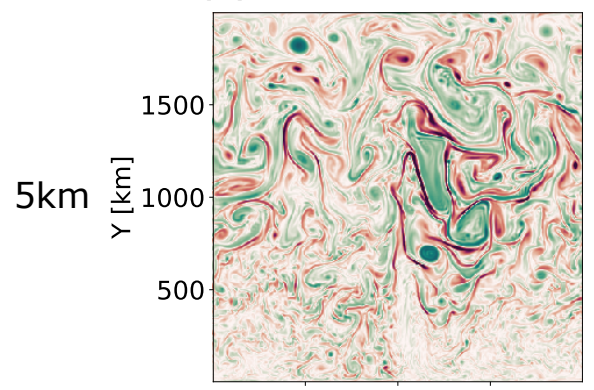

(g)

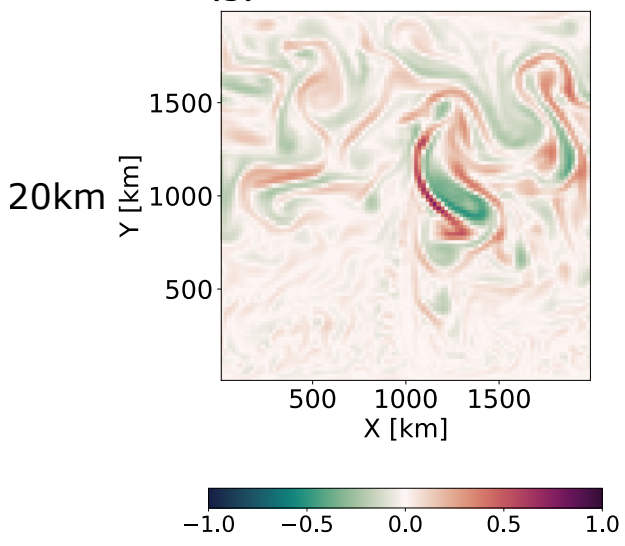

(b) $\quad \sigma /\left|f_{0}\right|$

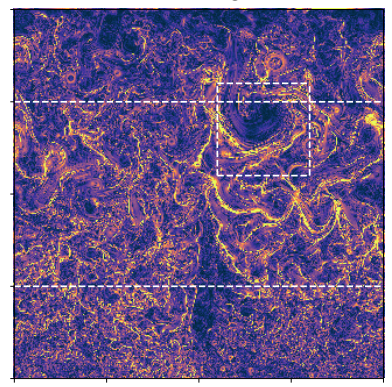

(e)

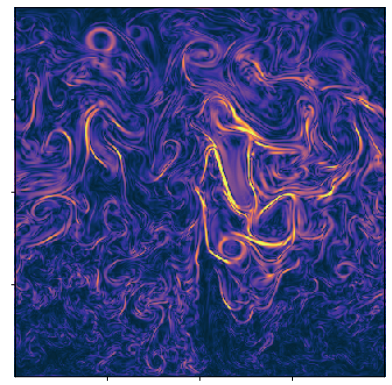

(h)
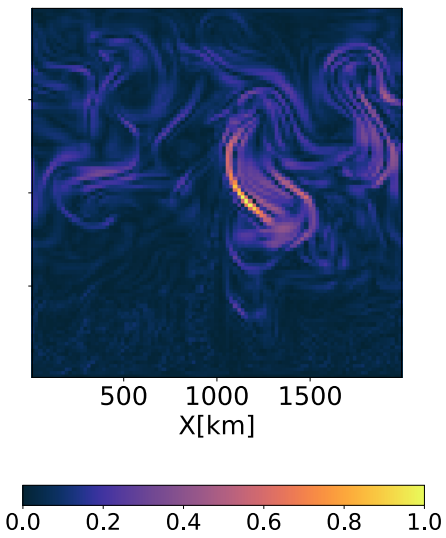

(c) $\mathrm{C}\left[\mathrm{kg} / \mathrm{m}^{3}\right]$

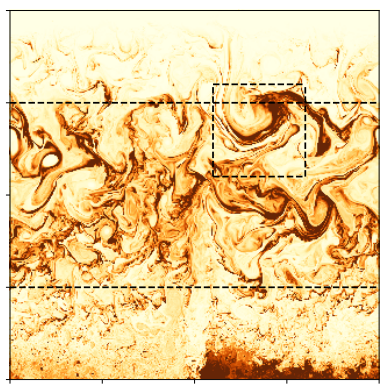

(f)

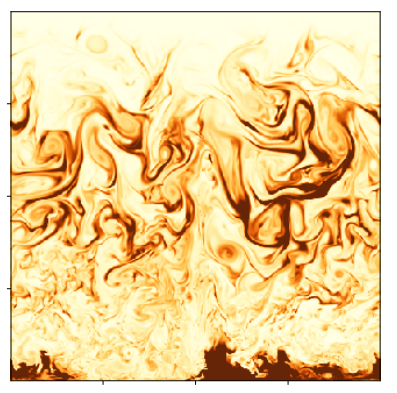

(i)

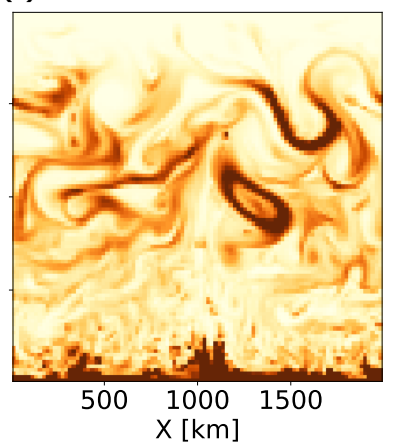

$\begin{array}{llllll}0.0 & 0.2 & 0.4 & 0.6 & 0.8 & 1.0\end{array}$

FIG. 2. Snapshot of surface vorticity $(\mathrm{a}, \mathrm{d}, \mathrm{g})$, surface strain $(\mathrm{b}, \mathrm{e}, \mathrm{h})$, tracer concentration at base of mixed layer (c,f,i) at $1 \mathrm{~km}$ (top row), $5 \mathrm{~km}$ (middle row) and $20 \mathrm{~km}$ (bottom row) resolutions. The vorticity and strain are normalized by the Coriolis frequency. The snapshot are taken 4 months after the tracer forcing is turned on. The horizontal dashed lines at $y=500$ and $1500 \mathrm{~km}$ in the upper left figure encompass the analysis region used for most of the diagnostics in this study, and the dashed box downstream of the ridge indicates the region that is used for the fields in Figure 4. 


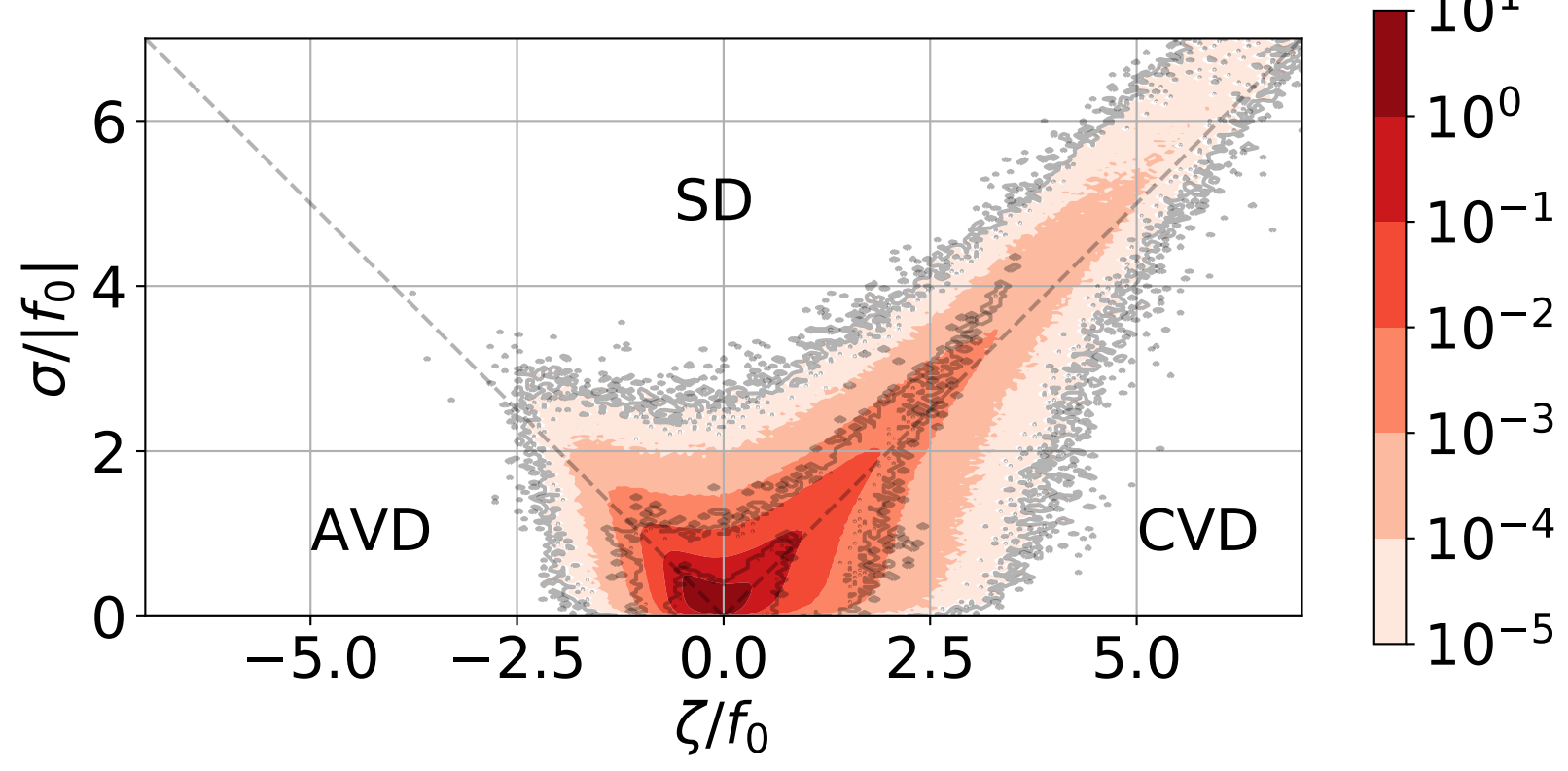

FIG. 3. Surface vorticity-strain JPDF for the $1 \mathrm{~km}$ simulation. The gray contour lines correspond to the outer limits $\left(P(\zeta, \sigma)=10^{-5}\right)$ for the JPDFs from the $1 \mathrm{~km}, 5 \mathrm{~km}$ and $20 \mathrm{~km}$ simulations, with the innermost contour corresponding to the lowest resolution and the outermost contour corresponding to the highest resolution. In this and all following plots on the $\zeta / f_{0}$ vs $\sigma /\left|f_{0}\right|$ plane, the dashed lines are the $\sigma=|\zeta|$ lines. These lines demarcate the boundaries between the strain dominated (SD), anticyclonic vorticity dominated (AVD), and cyclonic vorticity dominated (CVD) regions. 


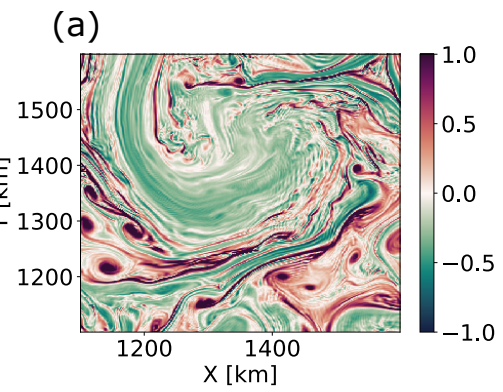

(d)

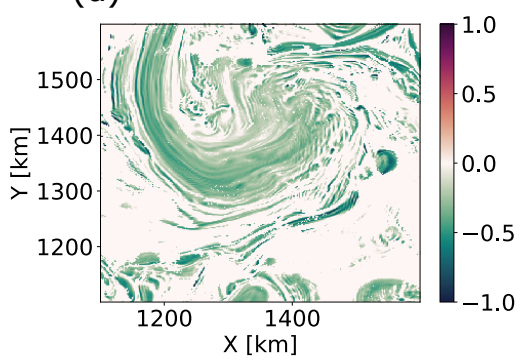

(b)

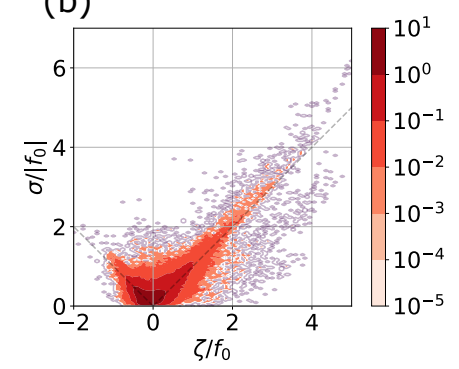

(e)

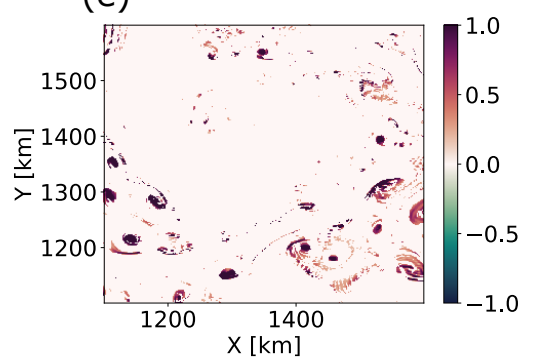

(c)

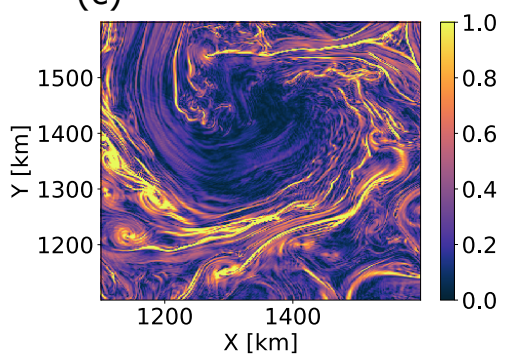

(f)

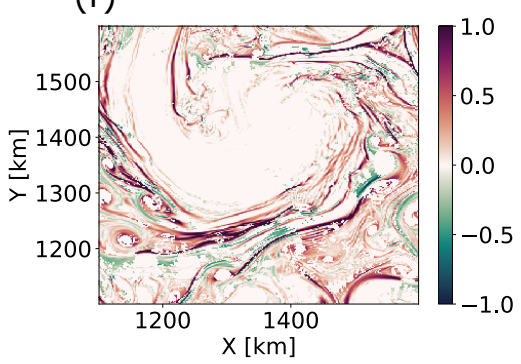

FIG. 4. Surface vorticity-strain based flow decomposition. Surface vorticity (a) and surface strain (c) in a large scale anticyclonic meander downstream (dashed box in Figure 2). (b) The JPDF corresponding to this region. Bottom row shows the surface vorticity decomposed based on where the grid points lie in the JPDF; corresponding to the AVD (d), CVD (e), and SD (f) regions. 

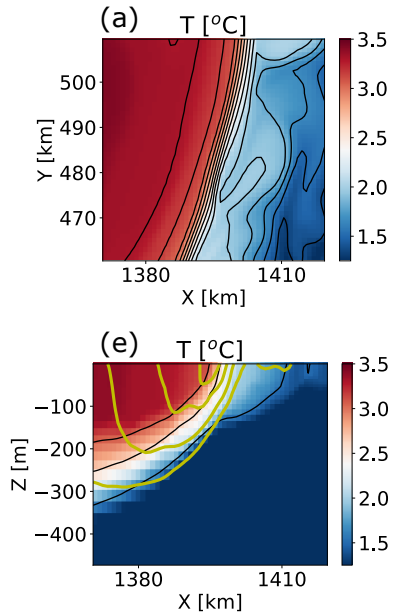

(b)
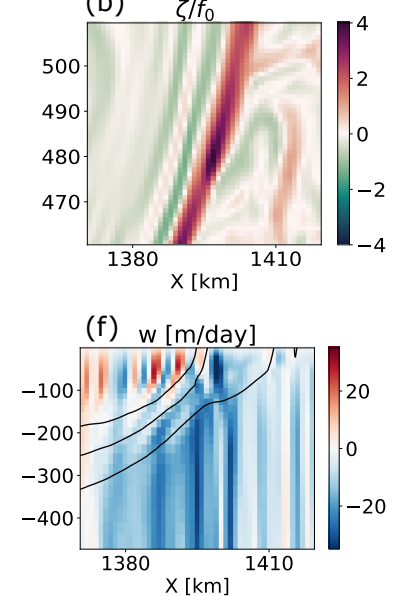
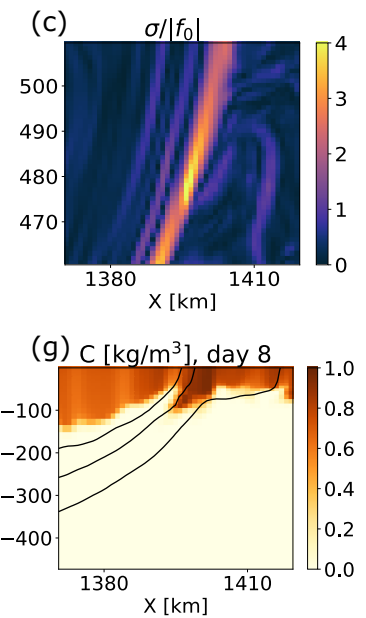
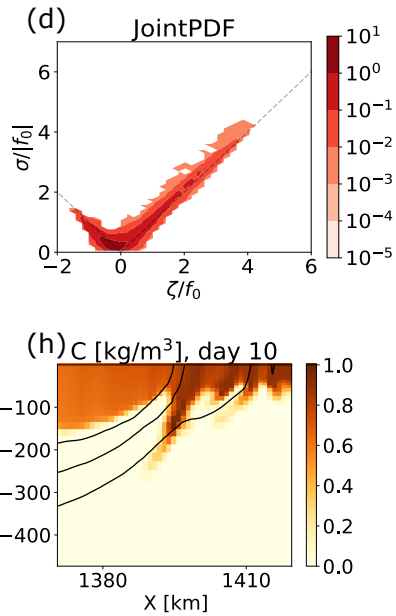

FIG. 5. Properties of a typical front. The surface temperature (a), surface vorticity (b), surface strain (c), and the vorticity-strain JPDF (d) in a region with a strong front. A depth-across front section of the temperature (e), vertical velocity (f), and tracer on day $8(\mathrm{~g})$ and $10(\mathrm{~h})$ after the tracer forcing is turned on. The black contours in (a, e, f, g, h) are some chosen temperature contours to highlight the front. The yellow contours in (e) show the meridional velocity, which is northwards, decaying away from the front. 

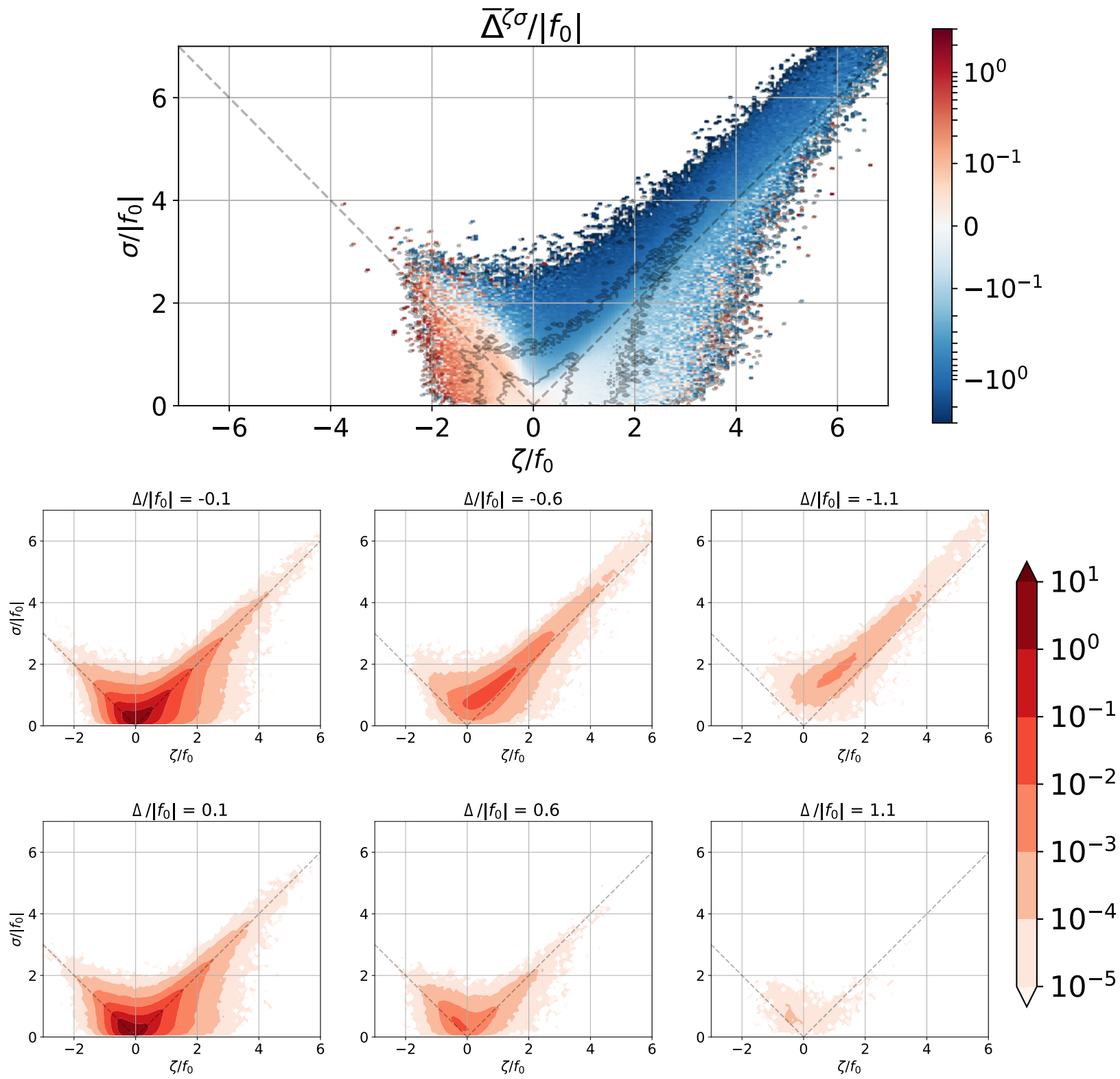

FIG. 6. Relationship of surface divergence to vorticity and strain. (top panel) The mean surface divergence conditioned on surface vorticity-strain. (bottom two rows) Slices of the 3D vorticity-strain-divergence JPDF at particular values of surface divergence (as indicated in panel titles); top row corresponds to convergent regions and bottom row to divergent regions. The gray contours and dashed lines are the same as in Figure 3. 

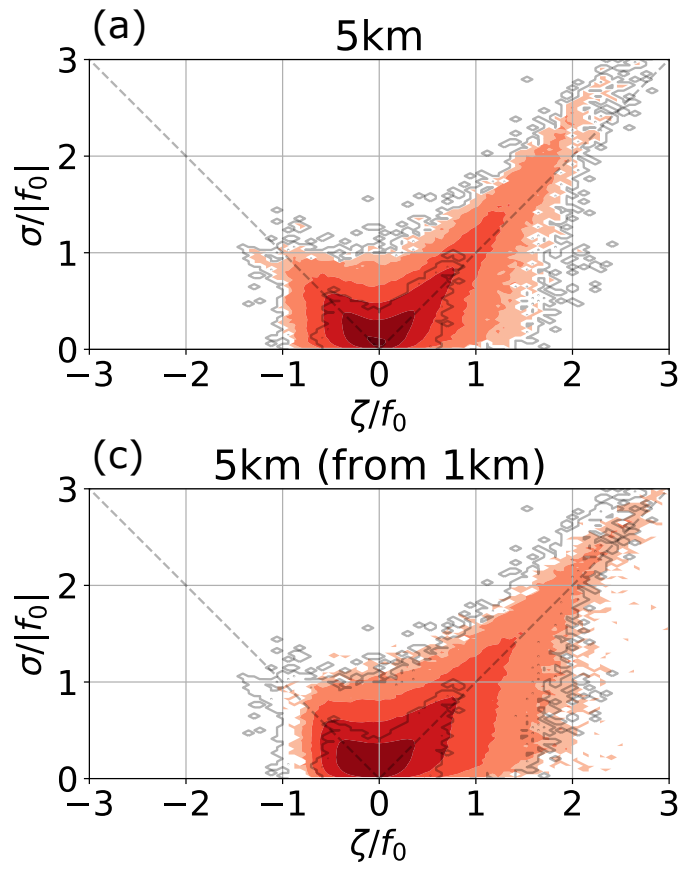

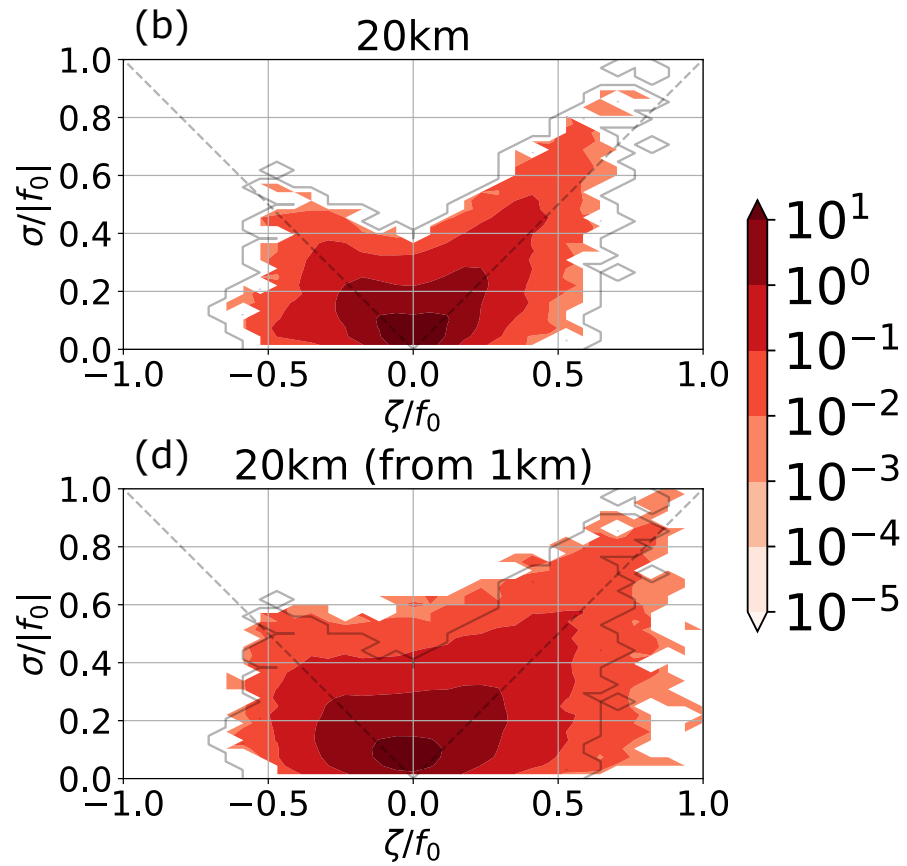

FIG. 7. Surface vorticity-strain JPDFs for the $5 \mathrm{~km}$ (a) and $20 \mathrm{~km}$ (b) simulation, and for the coarse-grained velocities from the $1 \mathrm{~km}$ simulation to $5 \mathrm{~km}$ (c) and $20 \mathrm{~km}$ (d) respectively. The gray contours and dashed lines are the same as in Figure 3. Note the changing axis limits with resolution, and relative to Figure 3. 
(a) middle of mixed layer
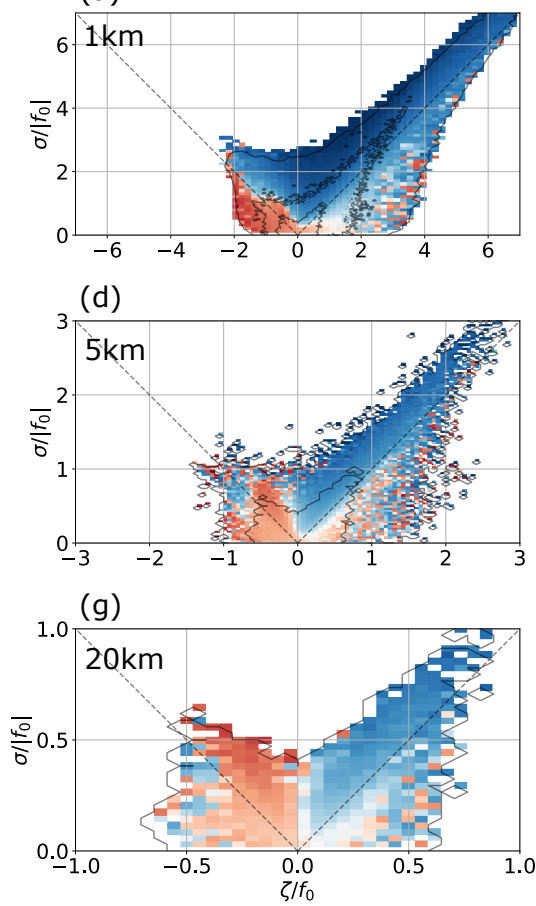

(b) bottom of mixed layer
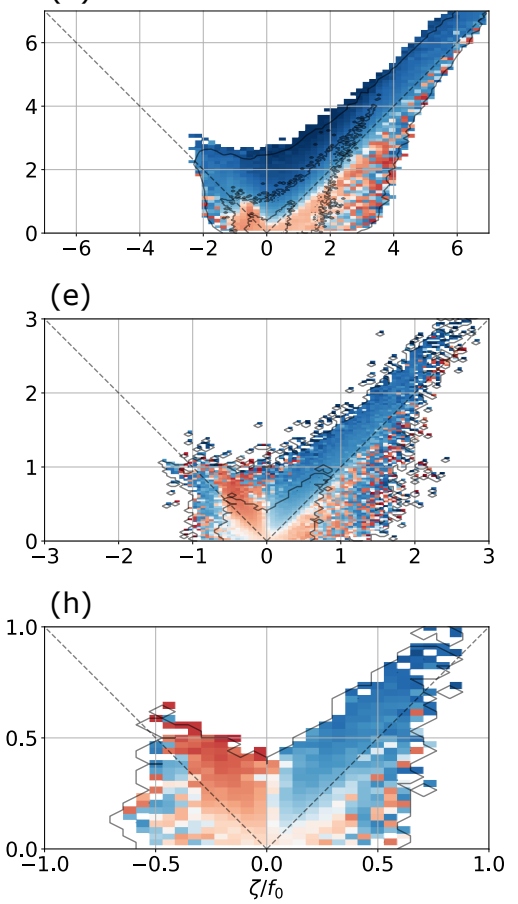

(c)
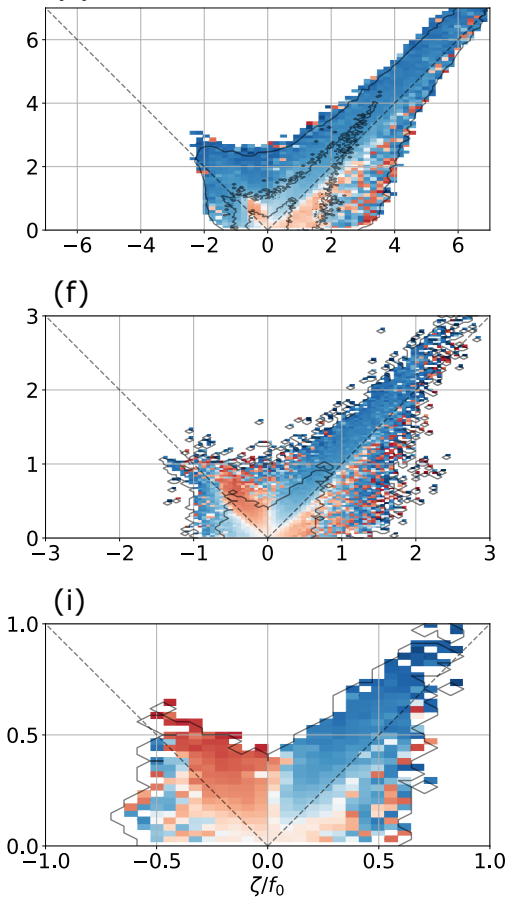

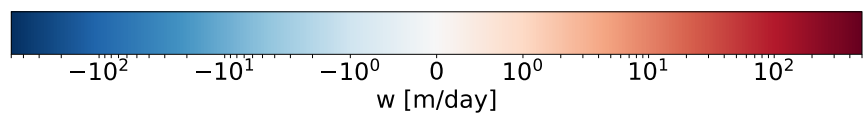

FIG. 8. Conditional mean of the vertical velocity conditioned on the surface vorticity and strain at different resolutions (columns) and depths (rows). The top row is for $1 \mathrm{~km}$ resolution, followed by the $5 \mathrm{~km}$ and then the $20 \mathrm{~km}$. The first column corresponds to the middle of the mixed layer $(50 \mathrm{~m}$ for $1 \mathrm{~km}, 75 \mathrm{~m}$ for $5 \mathrm{~km}$ and $90 \mathrm{~m}$ for $20 \mathrm{~km}$ ), the second column to the base of the mixed layer (100m for $1 \mathrm{~km}, 150 \mathrm{~m}$ for $5 \mathrm{~km}$ and $180 \mathrm{~m}$ for $20 \mathrm{~km}$ ), and the third column to a fixed depth of $250 \mathrm{~m}$. The gray contours and dashed lines are the same as in Figure 3. Note the changing axis limits with resolution. 

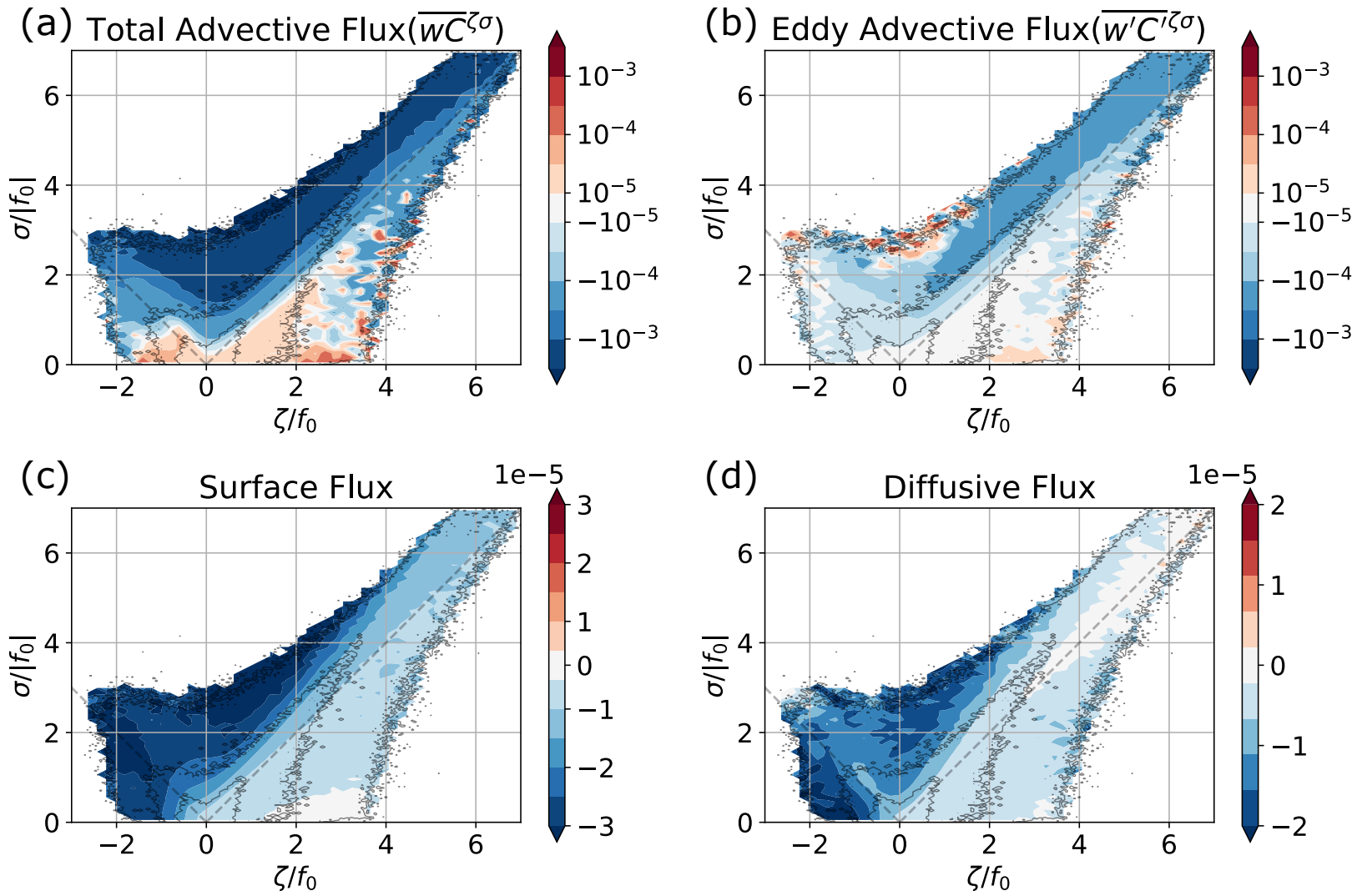

FIG. 9. Conditional mean of different components of the tracer flux conditioned on the surface vorticity and strain; the components being (a) the total advective flux, (b) the eddy advective flux, (c) the surface flux, and (d) the diffusive flux. Notice that the different panels are for different depths and have different color ranges, the color ranges were chosen to allow a comparison across different regions of the JPDF rather than across figure panels. The diffusive flux is computed at the depth of $50 \mathrm{~m}$, which is the middle of the mixed layer - where the parameterized KPP boundary layer diffusivity is the highest (not shown), and the advective fluxes are computed at the depth of $100 \mathrm{~m}$, which is the base of the mixed layer. The gray contours and dashed lines are the same as in Figure 3. 

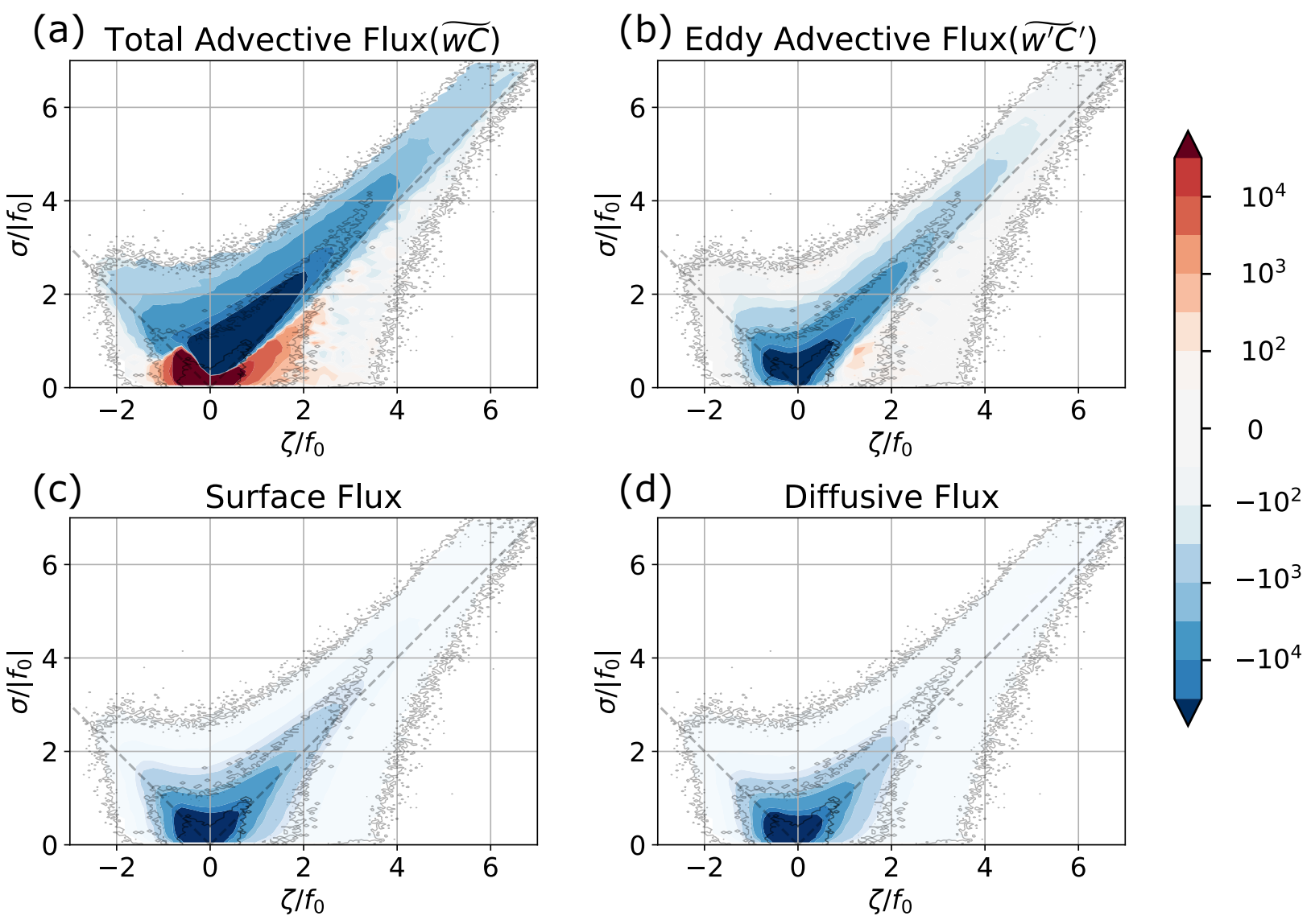

FIG. 10. The contribution of regions corresponding to different parts of the surface vorticity-strain JPDF to

tracer transport for the different components of the flux - (a) total advective flux, (b) eddy advective flux, (c) surface flux, and (d) diffusive flux. The gray contours and dashed lines are the same as in Figure 3. 

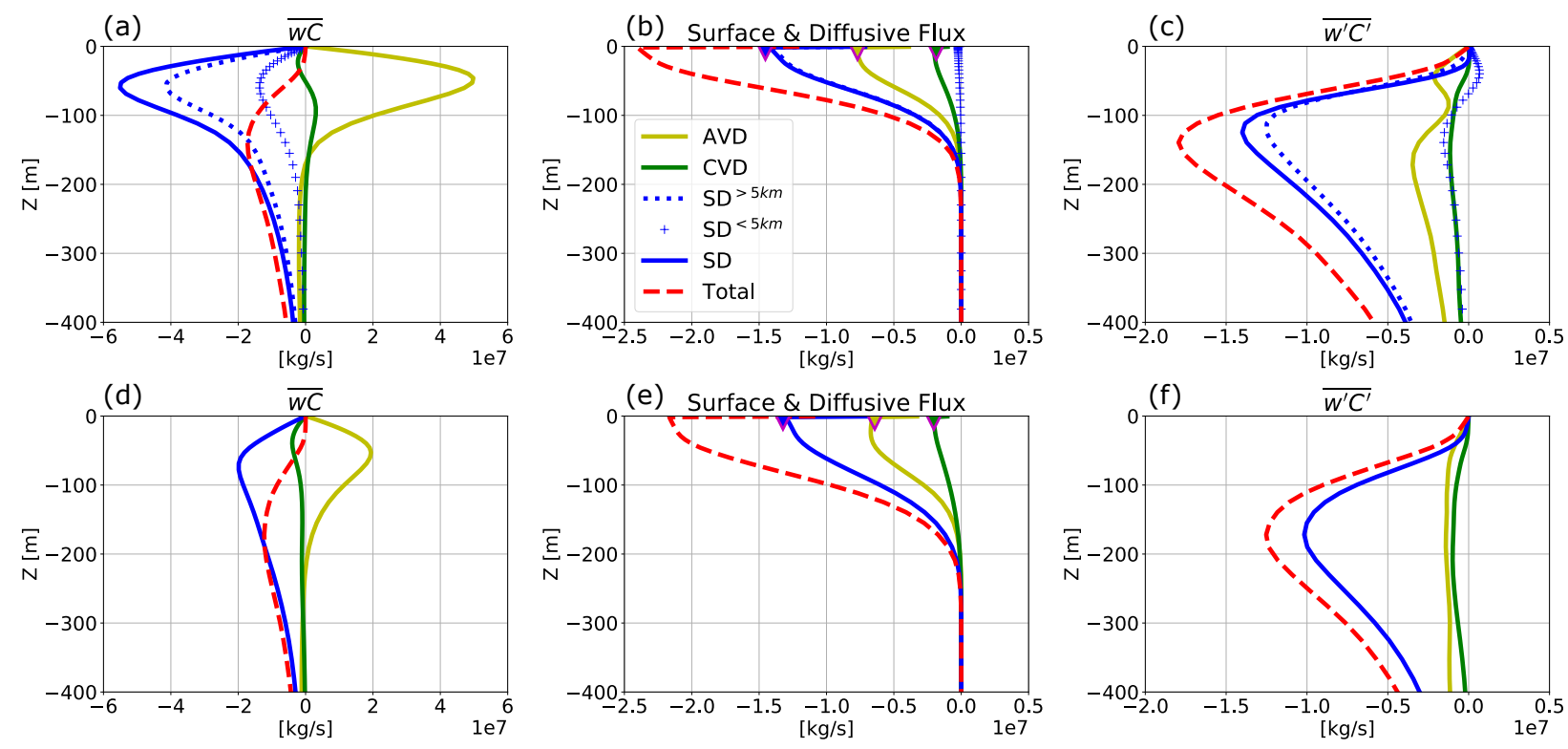

FIG. 11. Vertical structure of different tracer flux components in the $1 \mathrm{~km}$ (top row) and $5 \mathrm{~km}$ (bottom row) resolution simulations, separated into components based on the regions in the JPDF. The first column (a,d) shows the total advective flux $(\overline{w C})$; the second column $(\mathrm{b}, \mathrm{e})$ shows the diffusive flux and the surface flux (inverted red triangles); and the third column (c, f) shows the eddy advective flux $\left(\overline{w^{\prime} C^{\prime}}\right)$ integrated over the parts of the JPDF corresponding to SD, AVD and CVD region. The sum of the parts is shown as the dashed red line. For the $1 \mathrm{~km}$ simulation we have divided contribution from the $\mathrm{SD}$ region into $\mathrm{SD}^{>5 \mathrm{~km}}$ and $\mathrm{SD}^{<5 \mathrm{~km}}$ regions respectively. 
(a)

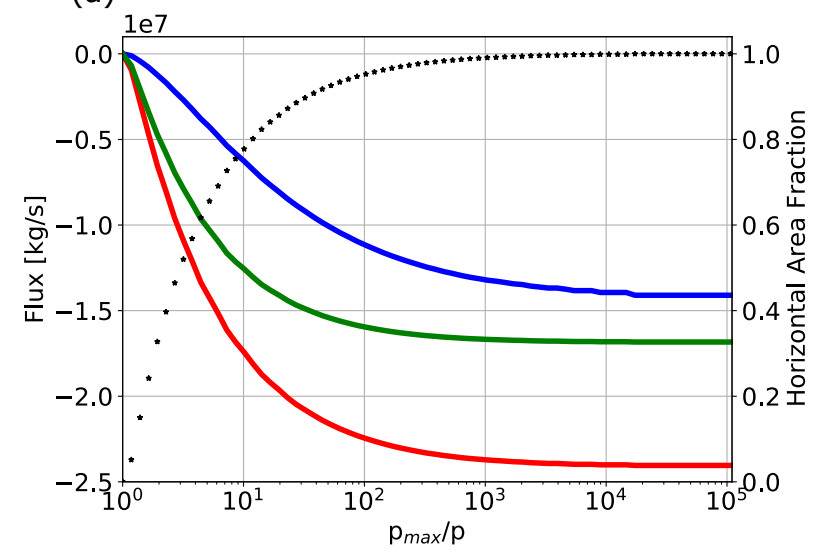

(b)

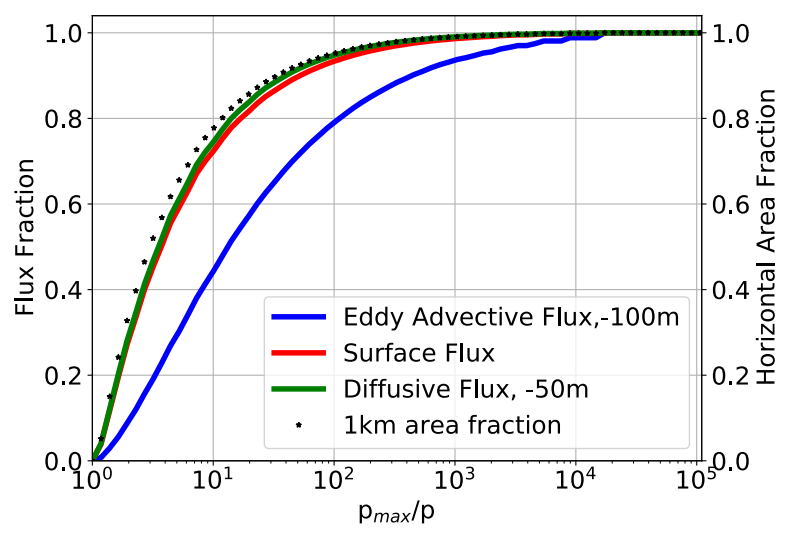

FIG. 12. Different flux components integrated outward from the maximum of the JPDF ( $\left.p_{\max }\right)$ is present to contours of decreasing probabilities $(p)$ in the surface vorticity-strain JPDF. The integral is plotted as a function of $p_{\max } / p$, where $p_{\max }$ is the probability at the maximum of the JPDF. As shown in section $2 \mathrm{c}$, higher values of $p_{\max } / p$ generally correspond to smaller-scale features, and thus the $\mathrm{x}$-axis in this plot serves as a rough proxy for scales. Each curve asymptotes to the respective total flux at the corresponding depth. (a) The eddy advective flux at $100 \mathrm{~m}$, surface flux, and diffusive fluxes at $50 \mathrm{~m}$ for the $1 \mathrm{~km}$ simulation. (b) The flux fraction, defined as the integrated flux divided by the total flux, for the different components shown in (a). The dotted black line (axis shown on right) corresponds to the spatial area fraction contained in the region corresponding to $p_{\max } / p$ for the $1 \mathrm{~km}$ simulation. 
(a) $1 \mathrm{e} 7$

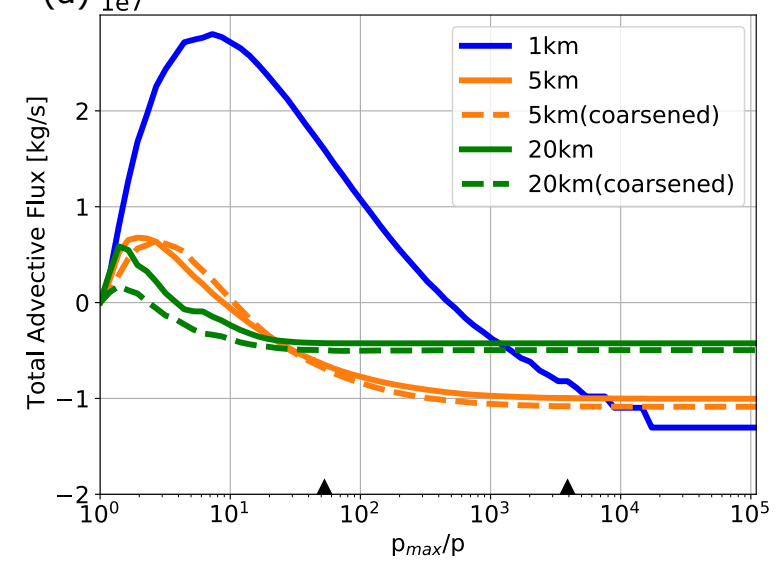

(b) $1 \mathrm{e} 7$

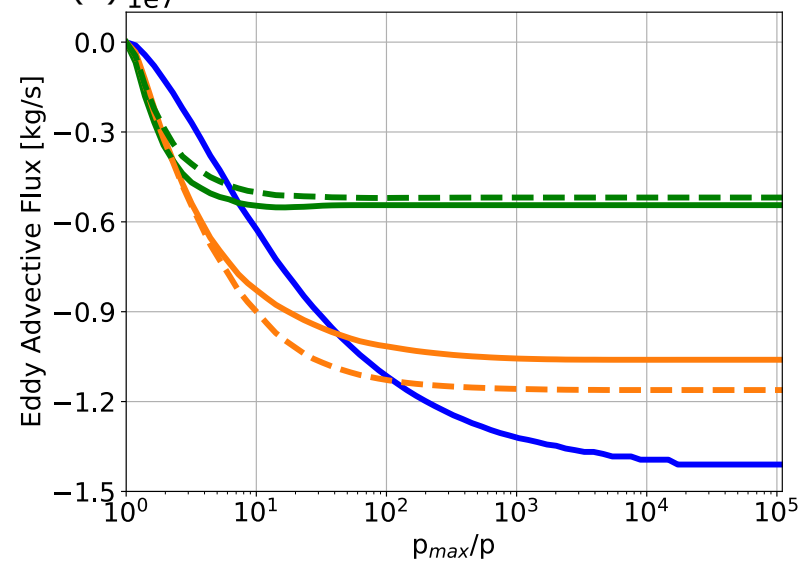

FIG. 13. The total advective flux (a) and the eddy advective flux (b) at the base of the mixed layer for different resolutions and also for different coarsening scales applied to the $1 \mathrm{~km}$ simulation. The $\mathrm{x}$-axis is the same as in Figure 12. Black markers at the bottom of (a) indicate roughly where the outer-most probability contours of $20 \mathrm{~km}$ and $5 \mathrm{~km}$ simulations lie relative to the $1 \mathrm{~km}$ JPDF. 

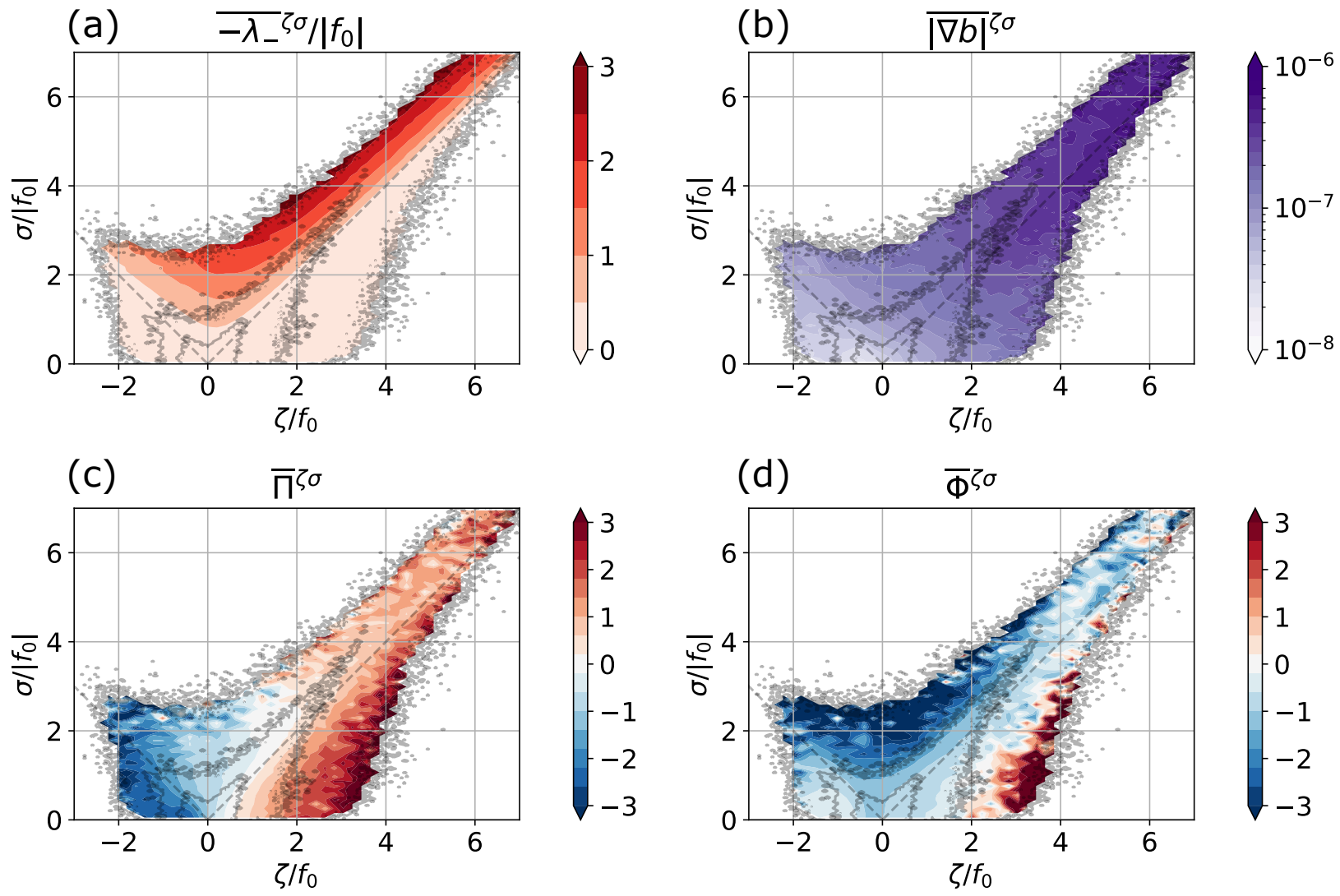

Fig. C1. Different criterion for instabilities and the strength of buoyancy gradients conditioned on vorticity-strain. The conditional means of (a) the normalized eigenvalue corresponding to rate of exponential growth of tracer gradients, (b) the absolute value of the buoyancy gradients, (c) the non-dimensionalized Ertel

PV ( $\Pi$, equation $\mathrm{C} 1)$, and (d) the generalized stability criterion ( $\Phi$, equation $\mathrm{C} 2)$ from Buckingham et al. (2021a). Negative values of $\Pi$ or $\Phi$ suggest potential for instability. The gray contours and dashed lines are the same as in Figure 3. 